\title{
A Survey on Computational Intelligence Approaches for Predictive Modeling in Prostate Cancer
}

\author{
Georgina Cosma ${ }^{\mathrm{a}, *}$, David Brown ${ }^{\mathrm{a}}$, Matthew Archer ${ }^{\mathrm{a}}$, Masood Khan ${ }^{\mathrm{b}}$, A. \\ Graham Pockley ${ }^{\mathrm{c}}$ \\ ${ }^{a}$ School of Science and Technology, Nottingham Trent University, Nottingham, UK. \\ ${ }^{b}$ University Hospitals Leicester NHS Trust, Leicester, UK \\ ${ }^{c}$ John van Geest Cancer Research Centre, Nottingham Trent University, Nottingham, UK
}

\begin{abstract}
Predictive modeling in medicine involves the development of computational models which are capable of analysing large amounts of data in order to predict healthcare outcomes for individual patients. Computational intelligence approaches are suitable when the data to be modelled are too complex for conventional statistical techniques to process quickly and efficiently. These advanced approaches are based on mathematical models that have been especially developed for dealing with the uncertainty and imprecision which is typically found in clinical and biological datasets. This paper provides a survey of recent work on computational intelligence approaches that have been applied to prostate cancer predictive modeling, and considers the challenges which need to be addressed. In particular, the paper considers a broad definition of computational intelligence which includes evolutionary algorithms (also known as metaheuristic optimisation, nature inspired optimisation algorithms), Artificial Neural Networks, Deep Learning, Fuzzy based approaches, and hybrids of these, as well as Bayesian based approaches, and Markov models. Metaheuristic optimisation approaches, such as the Ant Colony Optimisation, Particle Swarm Optimisation, and Artificial Immune Network have been utilised for optimis-
\end{abstract}

\footnotetext{
* Corresponding author

Email addresses: georgina.cosma@ntu.ac.uk (Georgina Cosma), david.brown@ntu.ac.uk (David Brown), m.archer1992@hotmail.co.uk (Matthew Archer), masood.khan@uhl-tr.nhs.uk (Masood Khan), graham.pockley@ntu.ac.uk (A. Graham Pockley)
}

Preprint submitted to Journal of $E^{A} T_{E} X$ Templates

November 4, 2016 
ing the performance of prostate cancer predictive models, and the suitability of these approaches are discussed.

Keywords: prostate cancer prediction; predictive modeling; computational intelligence; machine learning; soft computing; disease classification; evolutionary computation; metaheuristic optimisation.

\section{Introduction}

The increasing availability of electronic healthcare databases is enhancing opportunities for developing computer-based prediction and decision support models which can be used to improve the management of patients by healthcare 5 professionals. An important challenge for clinical teams remains the prediction and assessment of risk, and the development of accurate approaches for diagnosing, and predicting the diagnosis and therapeutic responsiveness and outcomes. The aim of predictive modeling in the context of medicine involves the development of computational models which are capable of predicting future events and/or healthcare-related outcomes for patients using contemporarily-available healthcare data (Waljee et al., 2014), (Shariat et al., 2009b). These models can be based on statistical techniques or computational intelligence techniques, with the latter being a relatively new strategy.

Computational intelligence approaches combine evolutionary algorithms such as the Genetic Algorithm and Particle Swarm optimisation, with machine learning algorithms such as the Deep Learning, Support Vector Machines, Bayesian models, and hybrids of these (Mumford \& Jain, 2009) for optimising the performance of the prediction model. Machine learning algorithms have a fundamental role in predictive modeling, as they can be utilised to create the component which learns from existing patient data in order to be able to make predictions on new patient data. Take, for example, a model which has been developed to predict prostate cancer. Given a set of inputs (also called features, predictors, variables, observations) and a set of clinical results (also called targets), a model can be trained to learn the inputs of this dataset. Once the learning pro- 
cess is accomplished, then the model can accept new inputs and predict clinical outcomes.

Clinical prediction systems which consider a profile of variables for predicting an outcome require sophisticated computational methods (Tewari et al. 2001). Computational intelligence approaches have the capability to deal with

30 the imprecision and uncertainty which is typically apparent in clinical and biological data. Furthermore, these approaches are effective when the data to be modelled are too large or complex for conventional statistical techniques. For example, computational intelligence algorithms have been used in risk prediction models for breast cancer (Hameed \& Bagavandas, 2011), (Bourdes et al., 35 2007), cardiovascular disease (Vijaya et al. 2010), and lung cancer (Sun et al. 2008), (Balachandran \& Anitha, 2013), (Diaz et al. 2014), (Dass et al., 2014), (Kumar et al. 2011).

This paper provides a survey of recent work on prostate cancer predictive modeling using computational intelligence approaches, provides a broader per40 spective of the area, and considers challenges that remain to be addressed.

The paper is structured as follows: Section 2 introduces the process of creating a classifier, cross validation techniques, and evaluation of classification models. Section 3 discusses the recent advances in computational intelligence algorithms that have been developed for prostate cancer, particularly the appli45 cation of Artificial Neural Networks, Deep Learning, Fuzzy approaches, Support Vector Machines, metaheuristic optimisation, Ensemble learning algorithms, and Bayesian approaches, including the Bayesian Network, and the Markov model. Section 4 discusses considerations for selecting a suitable metaheuristic optimisation method. Section 5 provides a discussion of advances, challenges and future areas of potential research. A conclusion and future directions are presented in Section 6 . 


\section{Cancer Classification and Evaluation}

Classification is the process of finding a model which is capable of distinguishing data records into classes. Prior to constructing a classification model, the dataset must be prepared using processes that may include the following:

- Data normalisation includes filling missing values, identifying and removing outliers, grouping variables, and normalising data.

- Feature extraction is the task of representing the original data in a reduced dimensional space. Feature extraction approaches are related to dimensionality reduction methods which include Principal Component Analysis (PCA) and Singular Value Decomposition (SVD).

- Feature selection involves selecting the most useful features without altering the original data representation, and hence choosing a subset of the features relevant for the task. In large datasets, evolutionary approaches such as the Genetic Algorithm can be used for finding the best subset of features.

Whether a feature extraction or selection approach is required depends on the type of data and the task. For example, feature extraction approaches are commonly applied to tasks involving image processing, for which it is important to represent the original image in a reduced dimensional space from which noise has been eliminated. On the other hand, feature selection is important for clinical data when the names of the features are important, for example when identifying which symptoms are the best predictors of a cancer; or when trying to identify which combination of features ('biomarkers') would make up the optimum cancer 'fingerprint.

A classification model can be constructed once the data preparation stage is completed. The process of constructing a classification model comprises of two main phases, as illustrated in Fig. 1 .

1. Learning phase (or training phase) in which the data are analysed using a classification algorithm, and from which a classifier (i.e. learned model) is 
created. During the learning phase, the classification algorithm analyses a set of training data which contains data records comprising of a set of inputs (also called features, predictors, variables, observations) and their known class labels (also called targets, known outputs) in order to learn from the data and build the classifier. A class label is the outcome of an event, for example cancer stage after diagnosis. The class labels are only used during the training process in order to enable the classifier to reason with the inputs. It should be noted that, since the class labels are provided to the classifier during the learning process, this phase is also known as supervised learning. Supervised learning is different from unsupervised learning (or clustering), during which the class label of each data record is not known in advance. Once a classifier is trained, a classification model is derived, and that model is used to predict the class labels of data records for which the class labels are unknown. The classification model may be represented in the form of neural networks, classification rules, graphical models such as Bayesian networks, Markov random fields and decision trees, or as a mathematical/statistical formulae (Han et al., 2011). Fig. 1 provides an overview of the process of creating a classification model for predicting patient outputs (i.e. class labels); and

2. Prediction phase (or testing and validation) of the model is used for classification/prediction tasks. During this phase, the learned model classifies new unknown data (contained in test and validation datasets) which have not been previously seen by the model in order to evaluate the accuracy of the learned model. Initially, the predictive accuracy of the model is evaluated and, if it is acceptable, then the learned model can be used for prediction.

Studies show that the accuracy of the classifiers depends on the specific dataset and the problem to be solved, and that there is no single classifier which outperforms all other classifiers across all tasks. Furthermore, the choice of validation method influences the reported accuracy of the classifier. The 


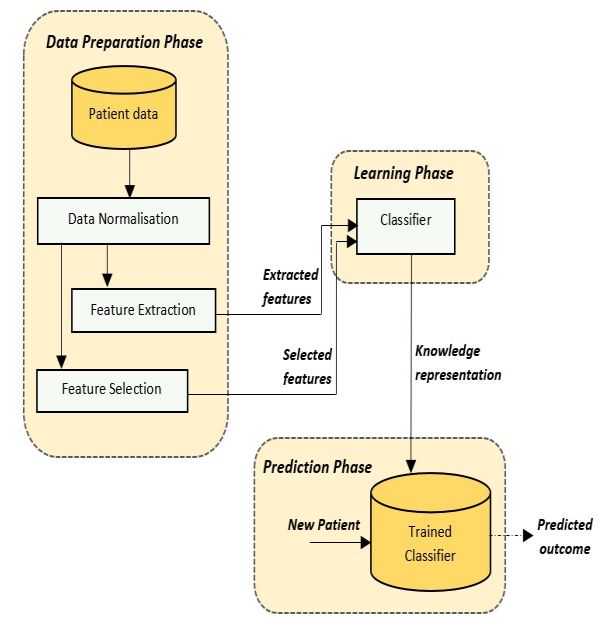

Figure 1: Process of creating a predictive model: Initially the dataset is prepared and a classification model is constructed. At the Learning Phase, the classifier learns from inputs (e.g. patient data records) and known class labels (known result). At the Prediction Phase, the classifier takes as input a new previously unseen records and predicts their class labels.

concept applies to clinical and biological data, and challenges related to sample size, especially when biological data is concerned (Swan et al., 2013).

There are a number of methods and metrics which can be used for evaluating the predictive performance of a classifier. Predictive models are most commonly evaluated using the cross-validation method discussed in Section 2.1, and a number of evaluation measures which are discussed in Section 2.2

\subsection{Cross-Validation}

Cross-validation is a technique for evaluating the performance of a model in terms of how well it performs on an independent dataset which was not used during the learning process. Cross-validation is also used for estimating how accurately a model will perform in clinical practice, and also for estimating how a selection of biomarkers can affect the outcome of a classification or prediction model.

As previously mentioned, in a typical prediction problem, a model is usually 125 trained on a given dataset and known class labels (training dataset), and tested 
on a previously unseen dataset of unknown data labels (testing dataset). Let A be an $m \times n$ matrix containing $m$ number of records (e.g. patient records), and $n$ number of variables (a.k.a. features, factors, or predictors). A record $X$ is represented by an $\mathrm{n}$-dimensional vector, $X=x_{1}, x_{2}, \ldots, x_{n}$, where $n$ is the total number of variables. Each vector, $X$, is given a known class label. In kfold cross-validation, the original dataset $A$ is partitioned into $k$ approximately equally sized subsets, $A_{1}, A_{2}, \ldots, A_{k}$. A single subset is retained for testing the model, and the remaining subsets are used for training the model. The cross-validation process is then repeated $k$ times (i.e. $k$ folds), with each of the $k$ subsets used exactly once as the validation data. Hence, in the first iteration subsets $A_{2}, \ldots, A_{k}$ are treated as the training set to create the first model, which is tested on subset $A_{1}$; the second iteration is trained on subsets $A_{1}, A_{3}, \ldots, A_{k}$ and tested on $A_{2}$; and the process continues. The accuracy is calculated by averaging the evaluation results obtained from each $k$ fold to produce a single estimation. Evaluation measures that are typically used are described in Section 2.2. The number of $\mathrm{k}$-folds is selected based on the size of the dataset, and usually ranges from 2 to 10 folds, but in general $k$ remains an unfixed parameter.

Two commonly used types of $k$-fold cross validation are employed in clinical research: leave-one-out and stratified cross-validation. With leave-one-out cross validation, one record is left out as the testing data and the remainder of the records are used as the training data - this process is repeated in every fold. Furthermore, in leave-one-out cross validation, the number of $k$ folds is equal to the number of records $m$, such that $k=m$; the training and testing process is repeated $m$ times, with a different record being left out for testing each time until all records are left out. With stratified cross-validation, the folds are stratified so that they contain approximately the same proportions of labels as the original dataset. (Kohavi, 1995) provides a comprehensive overview of cross-validation and other validation approaches for accuracy estimation and model selection.

Finally, it is important to adopt a cross validation methodology when constructing classification models in order to avoid overfitting the classification 
model to the trained dataset. Overfitting occurs when a model describes random error or noise instead of the underlying relationship, and can occur when a model is excessively complex by having too many parameters relative to the number of observations. A model will exhibit very good training performance and very poor predictive performance when overfitting occurs. Therefore, a suitable cross-validation should be adopted when evaluating computational intelligence models in order to ensure an efficient and more accurate reporting of the performance of the model.

\subsection{Evaluation of Classification models}

The performance of classification models in clinical tasks is often evaluated by the Receiver Operating Characteristic (ROC) curve analysis, an approach which is fundamental in clinical research. The ability of a system to differentiate between the data records in given classes (e.g. cancer patient or benign (no cancer disease) patient), is often measured by quantifying the ROC Area Under the Curve (AUC). The True Positive Rate (TPR, Sensitivity) measures the proportion of actual positives which are correctly identified as such (e.g. the percentage of cancer patients who were correctly identified as cancer patients), and the False Positive Rate (FPR, measured as 1-Specificity) measures the proportion of actual negatives which are incorrectly identified as positives (e.g. the percentage of benign patients who were incorrectly identified as cancer patients). The aim is to identify the optimal cutoff point which maximises the number of True Positives and minimises the number of False Positives on the ROC curve. This is often decided by the operator who is constructing the model.

The Area Under the Curve is a reflection of how good the system's performance is at discriminating between patients with and without disease, and the larger the Area Under the Curve the better the performance. Examples of ROC curves are presented in Fig. 2, which shows the results from evaluating the efficacy of four different types of tumour markers, as prostate cancer predictors, at various cutoff points (Chadha et al., 2014). 


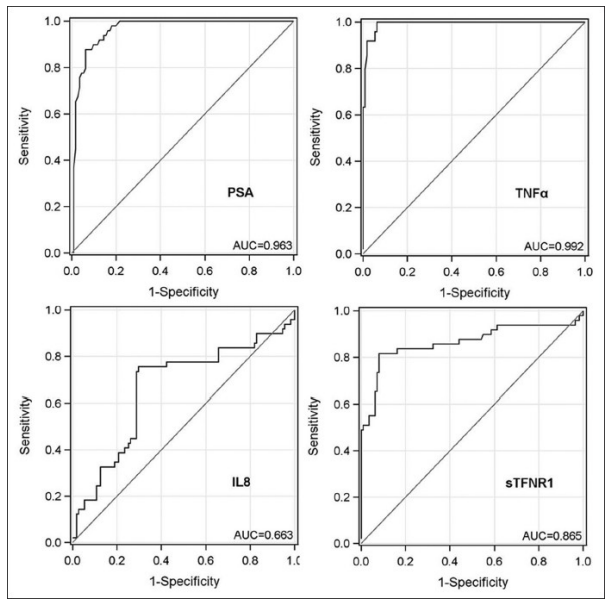

Figure 2: Receiver operating characteristic (ROC) graphical representation of the relationship between sensitivity and specificity. Each graph models the probability of local disease versus metastatic prostate cancer. Each ROC curve evaluates the efficacy of a tumour marker, as a prostate cancer predictor, at various cutoff points, and shows the Area Under the ROC curve. The figure shows that PSA (Prostate-Specific Antigen) and TNF (tumor necrosis factor- $\alpha$ ) levels were the strongest predictors of local versus metastatic disease. Soluble tumor necrosis factor- $\alpha$ receptor 1 (sTFNR1) was also a reasonably good predictor (Chadha et al. 2014). 


\section{Computational Intelligence Approaches to Prostate Cancer Pre- dictive Modeling}

Computational intelligence concerns the theory, design, application, and de-

190 which include Artificial Neural Networks, Genetic Algorithms, Evolutionary algorithms, Fuzzy systems, and hybrid intelligent systems. In contrast, computational intelligence is efficient in solving problems which require reasoning and decision-making and which traditional statistical models often fail to handle, mainly due to uncertainty, noise and dynamically changing data. This section describes the literature on computational intelligence approaches that have been applied to the prognosis and diagnosis of prostate cancer.

\subsection{Artificial Neural Networks}

Artificial Neural Networks are learning algorithms that are inspired by biological neural networks (the central nervous systems of the brain), and they consist of a set of interconnected input/output units in which each connection has an associated weight. The interconnected 'neurons' send messages to each other and their numeric weights can be tuned on the basis of the neural network's experience, thereby making neural networks adaptive to inputs and capable of makes it difficult to understand the qualitative reasoning behind the decisions that are made by the neural network. In addition, neural networks are more prone to overfitting than other computational intelligence algorithms and hence it is important to adopt a validation methodology, such as k-fold cross valida215 learning. During the learning phase, neural networks learn by adjusting their weights in order to predict the class label of the input records. Neural networks must be trained for long periods of time before they can be applied to solving problems such as classification or prediction. The limitation of the Artificial Neural Network is that it is difficult for humans to understand the symbolic meaning behind the learned weights of the hidden units in the network, which tion when reporting the performance of the neural network. The advantage of 
the Artificial Neural Network is that it often performs well with classifying data which have not been included in the learning process (hence data that have previously been unseen by the model).

\subsubsection{Artificial Neural Networks on Clinical Data}

Artificial Neural Networks have been applied to the early detection (Stephan et al. 2009) and diagnosis of prostate cancer (Djavan et al., 2002), and for the prediction of its outcome (Ecke et al., 2012). Neural networks are also effective for early diagnosis of prostate cancer when integrated into expert systems. The performance of the Artificial Neural Network has been compared to that of nomograms for detecting prostate cancer, and the vast majority of the literature supports the proposition that Artificial Neural Networks perform better than nomograms for risk prediction, and disease diagnosis and prognosis (Shariat et al. 2009a).

Interestingly, some studies have found that the predictive performance of the nomograms was close to that of the Artificial Neural Network, and that the performance of these models is dependent on the data and task in question. However, the outcomes of such studies were inconclusive. For example, Ecke et al. (2012) compared the performance of Artificial Neural Network against the nomograms developed by Karakiewicz et al. (2005) and Kawakami et al. (2008), and found that, during the testing phase, each of the methods performed very similar to each other, with no definitive answer to which is best. Consequently, they concluded that Artificial Neural Networks would be more successful for increasing detection rates, in daily practice, than nomograms. Artificial Neural Network classifiers have been applied for early diagnosis of prostate cancer and aiding the decision-making process without the need to perform a biopsy on patients. Çinar et al. (2009) applied an Artificial Neural Network and Support Vector Machines for early prostate cancer diagnosis on a reduced dataset (which comprised features: Prostate Specific Antigen (PSA), prostate volume, density, and smoking). To obtain the set of reduced features, they applied in245 dependent sample t-test statistical analysis as a technique for feature selection. 
The performance of the Artificial Neural Network was compared to that of the Support Vector Machine classifier, and the results revealed that the Artificial Neural Network and Support Vector Machine performances were very close. The performance of the Artificial Neural Networks reached its highest with $81 \%$

Machine reached its highest performance with $84.2 \%$ sensitivity, $74.8 \%$ specificity, and $81.1 \%$ accuracy, thereby slightly outperforming the neural network.

Similarly, Saritas et al. (2010) devised an Artificial Neural Network for the prognosis of cancer, the aim of which was to help doctors to decide whether definitively diagnosed with cancer after biopsy. Their results revealed an Area Under the Curve of $94.44 \%$, thereby suggesting that an Artificial Neural Network system can help doctors make quick and reliable diagnoses. However, these results appear to be inconclusive due to the very small dataset which was used and validation methodology adopted - 92 randomly selected records $(n=92$, $70 \%$ of the total data) were used for constructing the dataset used for training the Artificial Neural Network, and the remaining 29 data records ( $n=29,30 \%$ of the total data) were used for constructing the test dataset.

It is important to mention that when comparisons between prediction models, nomograms and other statistical models, are carried out, the same data and evaluation measures must be used for evaluating the predictive accuracy of the models in order to allow a fair comparison between them. This will reveal whether computational approaches are superior to nomograms when trained and tested on small datasets. Such a comparison is described in Cosma et al.

\subsubsection{Artificial Neural Networks on Images}

Artificial Neural Networks have been applied to the detection of prostate cancer using data obtained from transrectal ultrasonography and magnetic resonance images. In a study by Lee et al. (2006), the diagnostic performance of an 
transrectal ultrasonographic (TRUS) data which was obtained from 684 consecutive patients who had undergone TRUS-guided prostate biopsy. Their results revealed that performance improved when TRUS findings were included, with the ROC analysis revealing an average AUC of $85 \%$.

Artificial Neural Networks have also been successfully applied to determine the probability of malignancy by classifying information extracted from multiparametric Magnetic Reasonance Imaging (MRI) (Vos et al., 2012). When the performance of the Artificial Neural Network was compared to the systematic biopsy, the results revealed that it can achieve a lower false positive rate than systematic biopsy, and hence it can be utilised as a tool to assist radiologists and guide biopsies to the most aggressive location of the cancer. Using a similar application of neural networks, Matulewicz et al. (2014) have assessed whether an Artificial Neural Network can be utilised to detect cancer using information extracted from endorectal magnetic resonance imaging and magnetic resonance spectroscopic imaging (endo MRI/MRSI), and also information about anatomical segmentation. The Artificial Neural Network achieved a high accuracy with an Area Under the Curve, sensitivity and specificity of $96.8 \%, 62.5 \%$ and $99.0 \%$ respectively. Although the dataset used for performing the evaluations was very small and attributed to data availability, combining data obtained from images with information about anatomical segmentation is a promising way forward for improving detection rates.

A hybrid Neural Network and Support Vector Machine system has been embedded in a Computer-Aided Diagnosis (CAD) tool for predicting the Gleason Grade of prostate cancer using histopathology images (Greenblatt et al., 2013). This system uses the quaternion wavelet transform and modified local binary patterns for the analysis of image texture in regions of interest; and then utilises a two-stage classification method for predicting the Gleason grade. Firstly, a quaternion neural network with a new high-speed learning algorithm used for multi-class classification is applied; aftre which several binary Support Vector 305 Machine (SVM) classifiers are used for classification refinement. Experimental evaluations using the leave-one-out cross validation approach to predict the 
Gleason grades $(3,4$, and 5$)$ using a dataset of 71 images revealed a high degree of accuracy, $98.87 \%$, and also that it outperformed other published automatic Gleason grading systems. When results were averaged over all the classes, the system achieved a specificity rate of $99 \%$ and a sensitivity rate of $96.7 \%$. In the signal processing field, quaternions have been employed in adaptive filtering, including Kalman filtering (Choukroun et al., 2006) and stochastic gradient †ype of algorithm, such as the Quaternion Least Mean Square (QLMS) (Took \& Mandic, 2009).

\subsubsection{Deep Learning Networks}

Deep Learning is a sub-field of machine learning composed of models comprising multiple processing layers to learn representations of data with multiple levels of abstraction (Guo et al. 2016). Deep Learning models comprise multiple levels of distributed representations, with higher levels representing more abstract concepts (Bengio, 2013). Deep Learning algorithms are known for their capability to learn features more accurately than other machine learning algorithms, and are considered to be promising approaches for solving data analytics tasks with high degrees of accuracy. Recently, the Deep Neural Network, which is a variation of the standard Artificial Neural Network, has received attention. Many types of Deep Neural Networks exist, some of which are the Deep Boltzmann Machines (Salakhutdinov \& Hinton, 2009), the Restricted Deep Boltzmann machine (Hinton \& Sejnowski, 1986), and the Convolutional Deep Belief Network (Lee et al., 2009). These methods have dramatically improved state-of-the-art natural language processing (Mikolov et al., 2013), computer vision (Ciresan et al. 2012), as well as many other applications such as drug discovery and genomics (LeCun et al. 2015), and the analysis carcinoma images (Arevalo et al. 2015a). Convolutional neural networks have been applied for classifying mass lesions following mammography (Arevalo et al., 2015b). The Deep Boltzmann Machine has been applied for feature representation and fusion of multi-modal information from Magnetic Resonance Imaging (MRI) and Positron Emission Tomography (PET) for the diagnosis Alzheimer's Disease 
(AD) and Mild Cognitive Impairment (MCI) (Suk et al., 2014). The authors applied the system to solve three binary classification problems of $\mathrm{AD}$ versus healthy Normal Control (NC), MCI versus NC, and MCI converter versus MCI non-converter. Their results revealed that the system was highly accurate, with maximal accuracies of $95.35 \%, 85.67 \%$, and $74.58 \%$, respectively, thereby outperforming the competing methods. The authors concluded that the proposed Deep Learning method could hierarchically discover the complex latent patterns that are inherent in both MRI and PET. These findings highlight the potential value of Deep Learning on multi-modal neuro-imaging data for aiding clinical diagnosis. The application of Deep Learning algorithms to prostate cancer is starting to emerge. Guo et al. (2015) proposed a deep-learning based approach for the segmentation of the prostate using Magnetic Resonance (MR). Instead of training a classifier using handcrafted features, the authors have proposed a Deep Neural Network based approach which learns the feature hierarchy from the data. They found that the learned features were often more accurate in describing the underlying data than the handcrafted features.

Deep Belief networks are another type of Deep Learning Networks. Deep Belief networks are probabilistic generative models that are composed of multiple layers of stochastic, latent variables. Azizi et al. (2015) have developed an automatic feature selection framework for analysing temporal ultrasound signals of prostate tissue. The framework is based on a Deep Belief Network (DBN) model and consists of: an unsupervised feature reduction step that applies the model on spectral components of the temporal ultrasound data; and a supervised fine-tuning algorithm that uses the histopathology of the tissue samples to further optimize the model. Finally, a Support Vector Machine (SVM) classifier uses the activation of the Deep Belief Network as input to predict the likelihood of cancer.

\subsection{Fuzzy Approaches}

Traditional classification algorithms such as the Artificial Neural Network and the Support Vector Machine group each item into a single set (or class). In 
classical set theory, sets can wholly include or wholly exclude any given element (i.e. item). A fuzzy set is a set without a crisp or clearly defined boundary and can therefore contain elements with partial degrees of membership. Fuzzy

approaches add extra value to the prediction since they provide the reasoning behind the prediction in the form of rules. For example, they could provide 

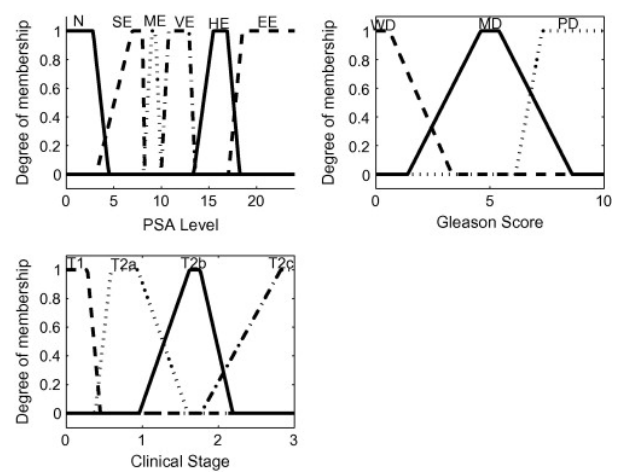

Figure 3: Membership functions which refer to input variables of PSA level, Gleason score, and Clinical stage (Castanho et al. 2013)

insight into which combinations of socio-demographic and early clinical features increase a patient's risk (useful for making predictions, and treatment decisions personalised to patients).

\subsubsection{Neuro-Fuzzy Approaches}

To date, fuzzy rule-based approaches are amongst the most popular approaches that have been applied to prostate cancer. Benecchi (2006) predicted the presence of prostate cancer using a co-active neuro-fuzzy inference system (CANFIS). CANFIS initially adopts a Genetic Algorithm (GA) to simultaneously search for features and thereafter uses the selected features to derive the fuzzy rules for prediction. Experiments were carried out with data from 1030 men, of which 195 (18.9\%) had prostate cancer. All men had total prostatespecific antigen (tPSA) level of less than $20 \mathrm{ng} / \mathrm{mL}$. Men with a Prostate Specific Antigen (PSA) level higher than $20 \mathrm{ng} / \mathrm{mL}$ in their bloodstream are likely (but not definitely) to have prostate cancer. The challenge in prostate cancer detection is to identify those men with prostate cancer who have PSA levels less than $20 \mathrm{ng} / \mathrm{mL}$. A PSA level higher than $20 \mathrm{ng} / \mathrm{mL}$ may occur due to factors other than cancer, such as urinary infection or an enlarged prostate. Their results revealed that the predictive accuracy of CANFIS was superior to that of the PSA blood test. Similarly, Keles et al. (2007) have created a Neuro-Fuzzy Clas- 
sification (NEFCLASS) tool for the diagnosis of prostate cancer, and prostate enlargement diseases. The symptoms of prostate cancer are very similar to those of prostate enlargement, and the aim of the classifier was to differentiate between these two scenarios and hence assign them to different classes. The authors only used data from 90 cases (and 47 values were missing from these cases), and concluded that the parameters of the NEFCLASS must be tuned for better prediction performance. However, results were inconclusive due to the small amount of data that were used to train and test the model.

Recently, Cosma et al. (2016) developed a neuro-fuzzy computational intelligence model for classifying and predicting the likelihood of a patient having Organ-Confined Disease (OCD) or Extra-Prostatic Disease (ED) using a prostate cancer patient dataset obtained from The Cancer Genome Atlas (TCGA) Research Network. The process for estimating the likelihood that the cancer has spread before treatment is given to the patient, is known as cancer staging prediction. Such a prediction is important for determining the most suitable treatment and optimal management strategy for patients. Clinical test results such as the pre-treatment Prostate-Specific Antigen (PSA) level, the most common tumor pattern in tumour tissue biopsies (Primary Gleason pattern) and the second most common tumor pattern (Secondary Gleason pattern), and the clinical T stage are commonly used by clinicians to predict the pathological stage of prostate cancer. The neuro-fuzzy system by Cosma et al. (2016) takes as input the results of those tests and an additional input, which is the age at diagnosis. Experiments revealed that the proposed neuro-fuzzy system outperformed other computational intelligence based approaches, namely the Artificial Neural Network, Fuzzy C-Means, Support Vector Machine, the Naive Bayes classifiers. The proposed neuro-fuzzy system also performed better than the AJCC pTNM Staging Nomogram (Edge et al. 2010) which is commonly used by clinicians. At its optimal point, the neuro-fuzzy system returned the largest Area Un445 der the ROC Curve (AUC), with a low number of false positives (FPR $=0.274$, $\mathrm{TPR}=0.789, \mathrm{AUC}=0.812$ ). The proposed approach is also an improvement over the AJCC pTNM Staging Nomogram $(\mathrm{FPR}=0.032$, TPR=0.197, $\mathrm{AUC}=0.582)$. 


\subsubsection{Genetic-Fuzzy Approaches}

n Using a different application of the fuzzy rule-based approach, Castanho ary Fuzzy Cognitive Map approach which is capable of learning Fuzzy Cognitive 
Maps. The proposed evolutionary Fuzzy Cognitive Map was applied to predict a patient's state after a period of time following a suggested therapy plan. The evolutionary Fuzzy Cognitive Map was applied to a small dataset comprising of 40 patients suffering with prostate cancer. The results revealed that the evolutionary Fuzzy Cognitive Map outperformed the basic Fuzzy Cognitive Map. Although it is difficult to determine the reliability of the results given the small dataset, it is important to appreciate that the authors gave appropriate thought to the validation of the results in order to ensure reliability of findings. They applied two methods to validation - method 1: the data were divided into learning and testing sets of the same cardinality (the learning set contained records of 20 patients, the remaining 20 records constituted the testing set); and method 2 : the leave-one-out cross validation (LOOCV) method was applied. The results revealed that both validation methods returned very similar results, and this may not have been the case if a larger dataset was utilised.

\subsection{Support Vector Machines}

Support Vector Machine (SVM) (Cortes \& Vapnik, 1995) is a method for the classification of linear and nonlinear data, and uses nonlinear mapping to transform the original training data into a higher dimension. Support Vector Machines then search for the linear optimal separating hyperplane, which essentially is a boundary which separates the records into classes. The Support Vector Machine finds the optimal hyperplane using support vectors (which can be characterised as the most significant training records) and margins (which are defined by the support vectors) (Han et al., 2011). The Support Vector Machine can be trained using several functions: the Linear Kernel Function, Quadratic, Gaussian Radial Basis (GRB), Multilayer Perceptron Kernel (MP) functions. Fig. 4 shows an example Support Vector Machine applied to ovarian cancer prediction using a linear separation of classes (Gaul et al., 2015). The most suitable training function is often experimentally selected. Support Vector Machines, when trained using the appropriate training function, are highly accurate and capable of modeling complex nonlinear decision boundaries (Han et al. 

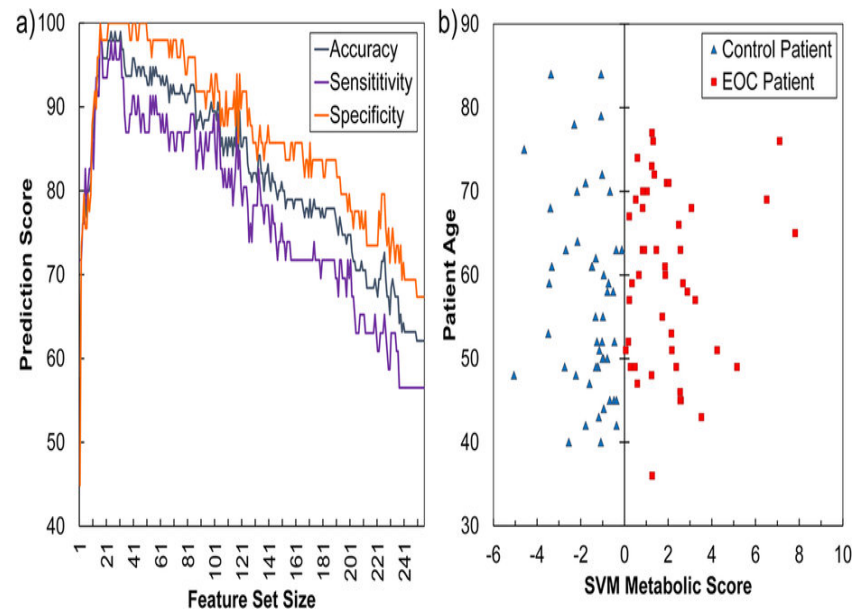

Figure 4: (a) Accuracy using Support Vector Machine (SVM) feature selection for metabolic detection of early stage ovarian cancer. Plot (a) shows the performance of the SVM based classifier against alternative approaches. A minimum of 16 metabolic features that provided $100 \%$ accuracy. Plot (b) shows the optimal linear separation between epithelial ovarian cancer (EOC) and control samples by the SVM-based model. The vertical line is the projection of the separating hyperplane generated by the SVM model. The discriminant linear SVM model was evaluated by leave-one-out cross-validation (LOOCV) (Gaul et al. 2015).

2011). They are also much less prone to overfitting than other computational intelligence methods, and can be used for classification as well as prediction tasks. Support Vector Machines have been adopted to solve decision-making tasks on data which also include data obtained from images, microarray and other clinical data.

The automatic identification of the boundary between the prostate and nonprostate regions is challenging, as the low contrast of ultrasound prostate images makes prostate and non-prostate boundaries fuzzy and noisy. Computational intelligence methods can be applied to identify and segment the prostate region on ultrasound images. Sung et al. (2011) proposed a Computer-Aided Diagnosis (CAD) system, based on Support Vector Machines, for prostate cancer detection using dynamic contrast-enhanced MRI (DCE-MRI) examinations. Their system was evaluated using DCE-MRI examinations of 42 patients, and the results revealed that the system achieved an overall accuracy, sensitivity and 
specificity of $83 \%, 77 \%$ and $77 \%$ respectively. In a recent study, Haq et al. (2015) also produced a DCE-MRI system for prostate cancer prediction. The approach by Haq et al. (2015) is data-driven and utilises a number of approaches for feature extraction and feature reduction. In particular, Principal Component Analysis (PCA) was applied along with the least absolute shrinkage and selection operator (LASSO) to extract the optimal set of principal components. Using the extracted features, a predictive model was created which was based on the Support Vector Machine classification algorithm. For testing the model, a total of 449 tissue regions were obtained from 16 patients, and the results revealed that the proposed system achieved an Area Under the Curve of $86 \%$ when using the leave-one-out cross validation approach.

Fei et al. (2011) have developed a PET/CT directed, 3D ultrasound imageguided biopsy system for improving the detection of prostate cancer. Their system was designed to automatically identify the areas of an image containing prostate and non-prostate tissues. The prostate textures were captured by training the locally placed Wavelet-based Support Vector Machines (W-SVMs). The trained W-SVMs were used to tentatively label the voxels around the surface into prostate and non-prostate tissues based on their texture features obtained from different Wavelet filters. Thereafter, boundaries were formed between the tentatively labelled prostate and non-prostate tissues based on defined weighting functions and labeled voxels. The authors found that, using local texture features and geometrical data, W-SVMs can improve the classifier's performance with regards to differentiating the prostate tissue from the adjacent tissues. They also found that TRUS image textures can provide important features for accurately defining the prostate, particularly for the regions where prostate boundaries are not clear. The system was evaluated using data sets containing 3-D TRUS images from 5 patients. The system achieved an average DICE overlap ratio of $90.7 \% \pm 2.5 \%$, and an average sensitivity of $90.7 \% \pm 4.9 \%$.

Fig. 5 shows sample segmentation and its comparison with the corresponding gold standard (obtained from manual segmentation) (Fei et al., 2011). Briefly, the DICE overlap ratio is the result of using the DICE coefficient (Dice, 1945) 


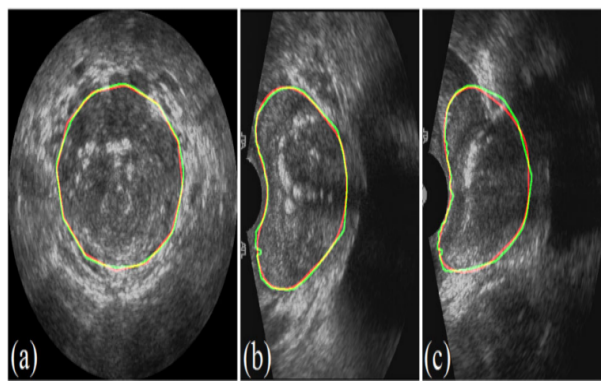

Figure 5: 2-D segmentation results in different planes: red lines are the gold standard boundaries (those defined by experts) and green lines are the segmentation boundaries (those defined by the system which uses Wavelet-based Support Vector Machines, W-SVMs). (a)Coronal plane (b) Sagital plave (c) Transverse plane (Fei et al. 2011)

which measures the extent of spatial overlap between two binary images. It is commonly used in reporting performance of segmentation and gives more weighting to instances where the two images agree. A DICE overlap ratio value ranges from 0 (or $0 \%$ ), indicating no spatial overlap between two sets of binary segmentation results, to 1 (or 100\%), indicating complete overlap and therefore perfect agreement. The performance of the system proposed by Fei et al. (2011) was tested on a very small dataset of images, and more evaluations need 560 to be conducted to conclude on the systems overall accuracy in automatically identifying the areas of an image containing prostate and non-prostate tissues.

Similarly, Kim \& Seo (2013) proposed an approach for segmenting prostate tissues using TRUS images and Gabor texture features, snake-like contour smoothing algorithm and the Support Vector Machine algorithm. Gabor feature extraction was applied to smooth the image and remove speckle noises, and the snake-like contour algorithm was then implemented to conclusively define the boundary of the prostate. After identifying the pixels defining the prostate boundary, the Support Vector Machine was trained using the pixels and known class labels (as identified by human experts) for classifying the pixels within prostate and non-prostate. During the testing phase, the accuracy of the algorithm in identifying the pixels defining the outline for the prostate boundary is compared to the results of a human expert who performed the same task. A 
total of 20 experimental images were used for the tests and the results revealed that the prostate boundaries generated differed from those that were drawn by human experts by only 10\%. More recently, a system proposed by Lehaire et al. (2014) used Support Vector Machines in combination with feature extraction for distinguishing between normal, normal but suspicious, and aggressive classes of cancer tissue found in multi-parametric magnetic resonance (mp-MR) images. The system was evaluated using mp-MR images of 35 patients. The system achieved a mean Area Under the Curve of $78 \% \pm 1 \%$, which compares well with other current state of the art systems.

Gertych et al. (2015) emphasise that although image classification approaches have been developed to identify and classify glandular regions in digital images of prostate tissues, the success of these has been limited by their capability to identify the different types of tissue components such as stroma, benign/normal epithelium and prostate cancer. They proposed a machine learning approach comprising of a Support Vector Machine followed by a Random Forest classifier to differentiate prostate tissue into stroma, benign/normal epithelium and prostate cancer areas. A total of 210 high resolution images of low-grade and high-grade tumors which were collected from 20 radical prostatectomies were manually annotated by two pathologists. The 210 images were split into the training $(n=19)$ and test $(n=191)$ sets. In the pre-processing steps, the Support Vector Machine separated the areas of stroma from the epithelium. Subsequently, areas of epithelium were stratified into benign/normal glands and prostate cancer using the Random Forest classifier. The Random Forest classifier was trained with a different number of trees. Algorithms were evaluated by Jaccard $(\mathrm{J})$, area overlap $(\mathrm{O})$ and Rand indices $(\mathrm{Ri})$ in order to determine the concordance of the algorithm-based prediction with pathologist manual annotations. $\mathrm{J}$ and $\mathrm{O}$ indices for concordance of stroma and epithelium areas were calculated separately, whereas Ri was calculated for stroma and epithelium together. To distinguish benign/normal glands from prostate cancer they trained a Random Forest classifier and obtained separate performance values for benign/normal glands and prostate cancer: $\mathrm{JBN}=35.2 \pm 24.9, \mathrm{OBN}=49.6 \pm 32$, 
$\mathrm{JPCa}=49.5 \pm 18.5, \mathrm{OPCa}=72.7 \pm 14.8$ and $\mathrm{Ri}=60.6 \pm 7.6$.

Singireddy et al. (2015) devised a Support Vector Machine classifier to identify biomarkers associated with prostate cancer progression (i.e. cancer stage) using RNA-Seq and the Support Vector Machine classifier. They utilised the Minimum Redundancy Maximum Relevance (mRMR) (Peng et al., 2005) statistical approach to select the best features, and thereafter a linear Support Vector Machine for performing the classification task. Although the reported accuracy was high, in the range of $80 \%$ to $99.3 \%$, this was a small-scale study which used a dataset of 106 prostate cancer samples. The authors do not report on the validation approach, nor whether the results reported were of the training or testing stage. Luque-Baena et al. (2014) performed a comparative study of the Stepwise Forward Selection (SFS) and Genetic Algorithms for the analysis of microarray data, with the aim of identifying groups of genes which have high predictive capability and biological relevance. They found that the predictive models returned higher accuracies when the Genetic Algorithm was utilised to select features as opposed to the Stepwise Forward Selection approach.

In summary, the automatic identification of the boundary between prostate and non-prostate continues to be challenging given that the boundary is often not clear in ultrasound prostate images and that their textures are difficult to classify. The identification and classification of cancerous and non-cancerous tissues by analysing image data is also a challenging and ongoing area of research. ${ }_{625}$ A common barrier relates to the availability of datasets which are large enough to ensure that the reported system performance is indeed representative. However, the studies conducted thus far in this area are yielding promising results. With regards to extracting relevant features from microarray data, this is also a very complex task because this type of data comprises a large number of features while few samples are generally available (Luque-Baena et al., 2014). The use of metaheuristic optimisation approaches for feature selection and boundary identification warrant examination, and the applications of metaheuristic optimisation algorithms to prostate cancer are discussed in Section 3.4 


\subsection{Metaheuristic Optimisation}

635 sation problems and are applied to find, generate, or select a heuristic (partial search algorithm) that may provide a sufficiently good solution to a problem. Metaheuristic algorithms are especially effective when the information is uncertain and dynamic (Bianchi et al., 2009). Some of the most popular metaheuristion (Kennedy \& Eberhart, 1995), Artificial Bee Colony algorithm (Teodorovic et al. 2006), and Ant Colony optimisation algorithm (ACO) (Dorigo \& Gambardella, 1997). Many of these nature-inspired algorithms are developed by studying the evolutionary behaviour of species (e.g. birds, insects, humans) and mimicking this in a computer science algorithm. Metaheuristic optimisation approaches are most commonly used for finding the best features in large datasets. These approaches require their parameters to be carefully tuned using suitable parameter values which need to be adapted based on the dataset and problem to be solved. Furthermore, models which comprise of a collection of optimisation and classification algorithms can be easily over-trained and extensive evaluations using appropriate validation approaches must therefore be carried out. In the subsections that follow, the metaheuristic optimisation approaches which have been applied to derive prostate cancer predictive models are discussed.

\subsubsection{Genetic Algorithm}

A Genetic Algorithm is an evolutionary algorithm (also known as a search heuristic or metaheuristic) which generates solutions to optimisation and search problems. Genetic Algorithms use techniques inspired by natural evolution, such as inheritance, mutation, selection, and crossover, and are often combined with other computational intelligence approaches to solve classification and prediction problems. Underwood et al. (2012) developed a Genetic Algorithm based method that integrates a simulation model in a Genetic Algorithm with the aim of selecting the best Prostate Specific Antigen (PSA) test screening policy. Comparisons between the results obtained from their proposed method and previ- 
ously recommended screening policies revealed that patients should be screened more aggressively, but for a shorter length of time than previously published guidelines recommend. Their research clearly demonstrates how computational intelligence can be used to influence healthcare policies. Treatment planning for radiation therapy is a multi-objective optimisation process. Yu et al. (2000) present a machine intelligent scheme for the planning of radiation therapy which 670 is based on Multi-Objective Decision Analysis (MODA) and Genetic Algorithm (GA) optimisation. They found that a combination of MODA and GA optimisation can provide solutions to practical treatment planning tasks. The authors emphasise the potential for real time applications in radiotherapy. Shi et al. (2013) propose a new approach to segment prostate ultrasound images using the 675 Genetic Algorithm optimisation. Boundary curve representations are derived by using Principal Component Analysis (PCA), after which prostate boundary features are determined and a Genetic Algorithm applied in order to optimize the parameters of the implicit curve representation. Recently, McGeachy et al. (2015) developed a Simple Genetic Algorithm (SGA) for determining optimal seed distributions for low-dose-rate prostate brachytherapy (McGeachy et al. 2015). The SGA performance was evaluated by comparing solutions obtained from a commercial optimizer (Inverse Planning Simulated Annealing [IPSA]) with the same cohort of 45 patients. The results of the SCGA and the IPSA were similar, and clinically acceptable. The Genetic Algorithm has also been used in various hybrid models in which it has been used to optimise the performance of a classifier, or for feature selection tasks. For example, Castanho et al. (2013) proposed a hybrid rule-based fuzzy system for predicting the pathological stage of prostate cancer, which uses a Genetic Algorithm to tune the fuzzy rules and membership functions was discussed in Section 3.2.2. Other hybrid algorithms which use Genetic Algorithms are discussed in the relevant sections of the paper. 


\subsubsection{Ant Colony Optimisation}

\ In computer science, the Ant Colony optimisation algorithm (ACO) (Dorigo \& Gambardella, 1997) is a probabilistic technique for solving computational problems that are concerned with finding the best paths through graphs. Ant Colony optimisation algorithms are based on the behaviour of ants seeking a path between their colony and a source of food - ants search for, and mark, the best solutions and take account of previous markings to optimize their search. Ant Colony optimisation has been applied to solve a variety of issues, including classification (Martens et al., 2007) and data mining problems (Parpinelli et al. 2002). In the prostate cancer classification system proposed by Thangavel \& Manavalan (2014), Ant Colony Optimisation was applied to select the best features for detecting prostate cancer from TRUS images. Initially, the Regions of Interest (RoI) were identified from the TRUS images and thereafter optimisation approaches were applied to the selected Regions of Interest in order to find the best features. Rough set based algorithms (namely the Hybridization of rough set based Quick Reduct) were combined with the Ant Colony Optimisation (QR-ACO) algorithm for selecting the best features from the selected Regions of Interest of TRUS images in order to improve accuracy and efficiency (feature selection). As an alternative to QR-ACO, a Genetic Algorithm-Ant Colony Optimisation (GA-ACO) algorithm was also developed for reducing the feature set. Once the best features were selected, the Support Vector Machine was applied for classifying the images to benign and cancer. Their experimental results revealed that the Ant Colony optimisation algorithm is an efficient approach for selecting the minimal features that are required for highly accurate classification of cancer and benign images.

\subsubsection{Particle Swarm Optimisation}

The Particle Swarm Optimisation (PSO) approach (Kennedy \& Eberhart 1995) is an adaptive, randomly optimal algorithm which is constructed on swarm intelligence, as each particle within the swarm represents a possible solution to the optimisation problem. In a Particle Swarm Optimisation system, a particle 
is within the search space and, with each iteration, it adjusts its position on the basis of its own experience and that of nearby particles. The particles evaluate their own position on the basis of a fitness function, and the process is repeated of prostate cancer which also uses a two-stage fuzzy neural network. Regression analysis was initially performed to find out the important factors which affect prognosis, and these factors included age, Body Mass Index (BMI), Prostate Specific Antigen (PSA), Digital Rectal Examination (DRE), clinical T-stage, fuzzy neural network. The fuzzy neural network comprised two steps. During 
the first stage, clustering of the features was performed using the aiNET-K clustering algorithm which is based on an Artificial Immune Network Kuo et al., 2014) in order to find a good initial solution for the membership functions at the beginning of training. During the second stage, the Artificial Immune Network was integrated with Particle Swarm optimisation (called Opt-aiNET) for the training of a fuzzy neural network. Through iterative evolution, the algorithm tries to find the optimal solutions. The system was trained using a small dataset comprising of 100 records and using 10 -fold cross validation. Their experiments revealed that the proposed method has the potential to accurately predict prostate cancer, and that it outperformed the network-based fuzzy logic control system. The drawbacks of the proposed algorithm are computational time and parameter tuning.

\subsection{Ensemble Learning Approaches}

Ensemble learning is the process by which multiple models, such as classifiers, are strategically generated and combined to solve a particular problem. Ensemble learning algorithms are mainly used for classification and prediction problems and are considered to be effective in achieving high performance. An Ensemble method combines a number of $k$ learned base classifier models, $M_{1}, M_{2}, \ldots, M_{k}$, and aims to create an improved classification model, $M_{*}$, which is a combination of all the classifiers. A dataset, $A$, is decomposed into a total of $k$ training sets, such that $A_{1}, A_{2}, \ldots, A_{k}$, where all, but one datasets, $A_{i}(1 \leq i \leq k-1)$, are used to train each classifier $M_{i}$. The remaining dataset is used for validating the predictive accuracy of each classifier, and hence the 775 records found in this dataset have not been previously seen by any of the classification models. During the classification process, a record $X_{i}$ is passed to each classification model which then returns a vote (i.e. class label) for the given record. The Ensemble algorithm then returns a final class prediction which is based on the majority of the votes of the base classifiers (Han et al. 2011). Alternative Ensemble methods such as bagging, boosting and Random Forests have been created for deriving the final prediction (i.e. processing of multiple 
votes to reach a final prediction). The Ensemble approach described above is illustrated in Fig. 6.

Ensemble models reflect the real life decision making process where several opinions may be gathered, and then all opinions are considered to make a final decision. For example, individual opinions of several experts are typically taken into consideration before making a final decision and agreeing on a medical procedure to minimise errors and optimise patient treatment (Polikar, 2009).

The purpose of adopting, and ensemble approach which comprises several classifiers, as opposed to adopting a single classifier to solve a problem could be due to one or more of the following reasons (Polikar, 2009):

- Statistical reason: Ensemble algorithms are suitable when there is inadequate data to properly represent the data distribution;

- Computational reason: Ensemble models are chosen when there are many computational models which are suitable to solving a given problem, and there is uncertainty as to which model to apply for solving the problem; and

- Representational reason: certain problems are too difficult for a single/given classifier to solve, meaning that the decision boundary that separates data from different classes may be too complex for the classifier to learn. However, an appropriate combination of an Ensemble of classifiers may be capable of learning the boundary.

\subsubsection{Random Forests}

Random Forests (Breiman, 2001) are an Ensemble learning method that creates a number of decision trees using a random selection of attributes. Decision trees are a popular method for various machine learning tasks. Decision trees can grow to be very deep in order to be capable of learning irregular patterns. For this reason, they tend to over-fit their training datasets (and hence perform better during the training than during the prediction tasks) due to their 


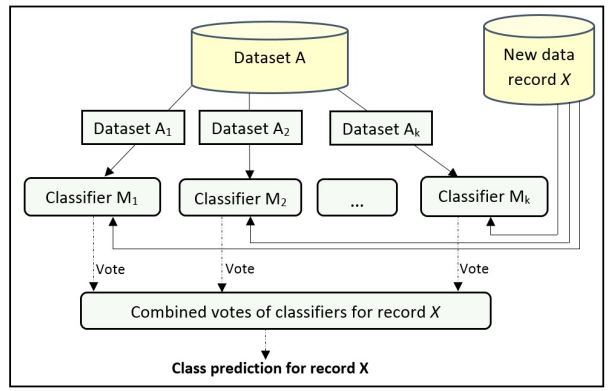

Figure 6: Ensemble classification: Combining an Ensemble of classifiers for making a prediction. A dataset is decomposed into $\mathrm{k}$ datasets which are used to generate a set of classification models, $M_{1}, \ldots, M_{k}$. A new data record is passed to each classifier, each classifier returns a vote (i.e. chosen class label). All votes are combined to return a final class prediction (i.e. class label). Adapted from Han et al. (2011). cancer radiotherapy (Ospina et al., 2014); to classify prostate cancer samples using a proteomic data set generated mass spectrometry data (Tong et al., 2004); and to identify biomarker panels in serum datasets for the detection and staging of prostate cancer (Fan et al. 2011). A recent application of Random Forest has ing the probability of prostate cancer. Their system is based on a combination of features which were extracted from a novel multi-parametric quantitative ultrasound elastography technique. The performance of the system was validated on 
a dataset containing data from 10 patients undergoing radical prostatectomy.

830 A Random Forest classification algorithm was applied to the combination of data extracted from the images in order to separate the cancerous regions from the non-cancerous regions, and to compute a probability for prostate cancer. During evaluations, the system achieved an Area Under the Curve of $82 \% \pm 1 \%$ using the leave-one-out cross validation, thereby demonstrating that this type 835 of system has potential for clinical use.

Another interesting application of Random Forests for the prediction of biochemical recurrence following prostate surgery has been proposed by Golugula et al. (2011). For this, multimodal data (imaging and non-imaging data) was collected and a hybrid approach combining Supervised Regularized Canonical ${ }_{840}$ Correlation Analysis (SRCCA) with a Random Forest classifier created. Their proposed approach was applied on a cohort of 19 patients, all of whom had radical prostatectomy, 10 of whom experienced biochemical recurrence within 5 years of surgery and 9 of whom did not. Their proposed approach reached a maximum classification accuracy of $93 \%$. Importantly, the authors emphasise that "computational challenges have limited the ability to quantitatively integrate imaging and non-imaging data channels with different dimensionality and scales"; few attempts have been made to mine such data for the purpose of constructing classifiers; and that no studies were found to "quantitatively combine histology (imaging) and proteomic (non-imaging) measurements for making diagnostic and prognostic predictions".

Frantzi et al. (2014) provide a very interesting discussion on biomarker research and how it is continuously expanding in the field of clinical proteomics. They discuss the technical proteomic platforms that are available along the different stages in biomarker discovery, and clinical applications relating to bladder cancer biomarker research. They emphasise that mass spectrometric techniques could provide highly valuable tools for biomarker research, and that advances could provide biomarkers that are clinically applicable for disease diagnosis and/or prognosis.

Much more research into combining non-image clinical data such as clini- 

at full (highest) resolution. A total of 10 image features are chosen from each resolution level through the use of an Adaboost Ensemble method. The system was tested on a set of 100 images, gathered from 58 patients, and evaluations 
revealed that it achieved at its lowest, intermediate, and highest levels an Area Under the Curve of $84 \%, 83 \%$, and $76 \%$ respectively. The authors found that their proposed boosted Bayesian multiresolution (BBMR) system outperformed both and individual features (no Ensemble) approach, and a Random Forest classifier Ensemble obtained by bagging multiple decision tree classifiers. Computerised image analysis is a challenge due to the size of the digitised histology images which contain hundreds of millions of pixels. Importantly, the authors found that different classes and types of image features become more relevant for discriminating between prostate cancer and areas of benign disease at different image resolutions. It was therefore important to extract features from different image resolutions (Doyle et al., 2012), as this improves image processing speed and system accuracy.

\subsection{Bayesian Approaches}

Bayesian classification is a probabilistic model which addresses the classification problem by learning the distribution of instances given different class values. This section describes the Naive Bayesian and the Bayesian Belief Network classifiers.

\subsubsection{Naive Bayesian Classifier}

Naive Bayesian classification is based on Bayesian theorem of posterior probability. Although it is designed for use when predictors within each class are independent of one another, it is known to work well even when that independence assumption is not valid. The Naive Bayesian approach, classifies data in two steps. The first step is the training (i.e. learning) step which uses the training input records to estimate the parameters of a probability distribution, assuming that predictors are conditionally independent. The second step is the prediction step, in which the classifier predicts any unseen test records and computes the posterior probability of that sample belonging to each class. It subsequently classifies the test records according to the largest posterior probability. Some of the functions that can be used for tuning the Naive Bayesian 
classifier include the Gaussian Distribution (i.e. normal distribution) and Kernel Density Estimation functions, and selecting which one to use depends on the dataset. Bayesian approaches have been applied to predict prostate cancer risk and risk of high-grade disease for men who undergo a prostate biopsy (Thompson et al., 2006). Strobl et al. (2015) demonstrated that a Bayesian approach integrated with Markov Chain Monte Carlo (MCMC) can improve the predictive performance of the well-known Prostate Cancer Risk Calculator (PCPTRC) tool, and also found that using Random Forests can consistently over-fit the training data. The Prostate Cancer Risk Calculator (PCPTRC) (Thompson et al., 2006) is a risk assessment tool that assesses patient risk, in consultation of their primary care physician.

930 Mazzetti et al. (2011) have used a Bayesian classifier for identifying malignant areas in Magnetic Resonance Images. In particular, they developed a Dynamic Contrast Enhanced Magnetic Resonance Imaging (DCE-MRI) ComputerAided Diagnosis (CAD) system to determine whether a given image area contains malignant tissue. The system can be used to help locate prostate cancer and guide biopsy to avoid unnecessary sampling. The system performs noise filtering and feature extraction. A Bayesian classifier is then applied to the extracted data to estimate the probability of malignancy. The dataset used by the authors was small and only included 10 patients with prostate cancer, and the evaluation results show that the system achieved an Area Under the Curve 940 of $89.9 \%$, a sensitivity of $82.4 \%$ and specificity of $82.1 \%$.

\subsubsection{Bayesian Network}

Bayesian networks are probabilistic graphical models which model the dependencies among subsets of attributes (Lacave \& Dez, 2003). Bayesian networks are also known as belief networks, Bayesian Belief Networks and probabilistic networks. A Bayesian network consists of an acyclic directed graph, whose nodes represent random variables, and whose links represent relations among them, along with a probability distribution over its variables. The nodes can also correspond to actual variables given in the data or correspond to "hidden 
variables" which are believed to form a relationship. For example, in the case of ber of symptoms that could characterise a disease (Han et al. 2011). Bayesian Belief Networks are different to naive Bayes classifiers, in that Bayesian Belief Networks do not assume class conditional independence. Class conditional independence is when the value of a particular feature is independent of the value of 955 independence holds, then the Naive Bayes classifier is a more accurate approach than the Naive Bayesian approach.

Fig. 7] shows a simple belief network for six variables: FamilyHistory, LungCancer, PositiveXRay, Smoker, Emphysema, and Dyspnea. All six variables are casual knowledge about lung cancer, for example, people with FamilyHistory of lung cancer and who are smokers have a greater risk of lung cancer. The PositiveXRay variable is independent of whether a person has a FamilyHistory of lung cancer or is a Smoker. Since the outcome of PositiveXRay is known, then the results of the FamilyHistory or Smoker variables do not provide any additional information regarding PositiveXRay (Han et al., 2011). A Bayesian network provides a conditional probability table (CPT) for each variable. Fig. 7 shows the conditional probability table for the variable LungCancer, where the conditional probability for each known value of LungCancer is provided for 970 each possible combination of the value of its parents, for example,

$\mathrm{P}($ LungCancer $=$ yes $\mid$ FamilyHistory $=$ yes, Smoker $=$ yes $)=0.8$

$\mathrm{P}($ LungCancer $=$ no $\mid$ FamilyHistory $=$ no, Smoker $=$ no $)=0.9$.

Bayesian networks have been applied to the analysis of genomic data, and mainly for investigating relationships between data and different types of data 975 pbtained from multiple platforms in the context of prostate cancer. Xutao et al. (2007) created a Bayesian network approach which uses mass spectrometry and microarray data for cross-platform analysis of cancer biomarkers. Using their proposed Bayesian network model, they were able to identify fourteen genes/proteins as reliable serum biomarkers, including prostate specific antigen 


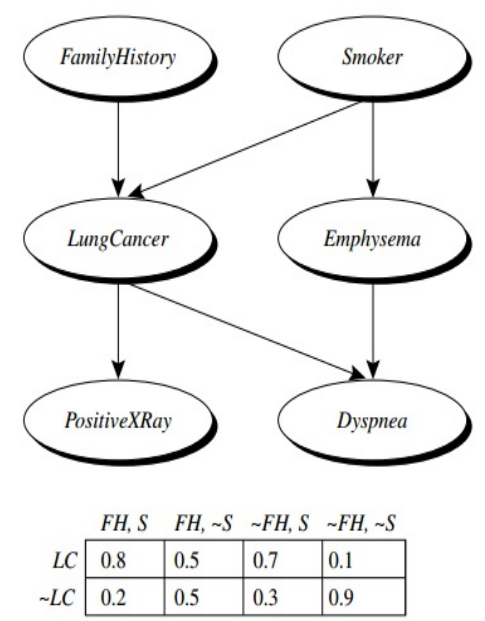

Figure 7: Simple Bayesian belief network. (a) A proposed causal model, represented by a directed acyclic graph. (b) The conditional probability table for the values of the variable LungCancer (LC) showing each possible combination of the values of its parent nodes, FamilyHistory (FH) and Smoker (S). Source: Adapted from Russell, Binder, Koller, and Kanazawa Russell et al. (1995) by Han et al. (2011).

(PSA). Importantly, the authors emphasise that combining different types of data reduces the number of putative biomarkers and allows for more-focused clinical studies.

Ni et al. (2014) developed a Bayesian network approach which can be used for investigating the relationships between genetic and epigenetic alterations, and how these mutations affect a patient's clinical outcome. They have applied their methods to identify known biologically relevant relationships and new genes that could potentially be novel biomarkers for cancer progression using a multiplatform dataset derived from patients with glioblastoma (an aggressive form of brain cancer). Jiang et al. (2014) have proposed a Bayesian network approach for patient survivorship prediction. The aim of the method is to predict patient survivorship, while having the capability to handle high-dimensional data and being able to incorporate it into a clinical decision support system (CDSS). Jiang et al. (2014) evaluated the system using a breast cancer dataset, and found that their proposed system outperformed the Cox proportional hazard 

image segmentation, and symbol detection or classification. A variation of the Pairwise Markov model (PMMs) has been applied to detect prostate cancer using information obtained from images. Monaco et al. (2009) proposed the 


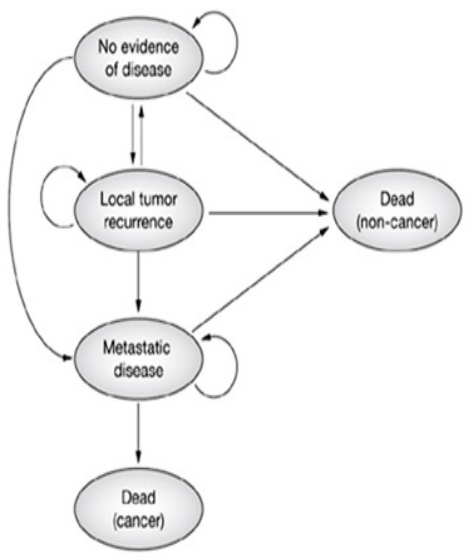

Figure 8: A Markov model of superficial bladder cancer. In each cycle, a patient cohort moves between the five health states. The direction of the arrows indicates the movement of the patients between the different health states (i.e. Markov states). Reflexive arrows (pointing back to their state) indicate that a patient can remain in a healthy state in successive cycles. Elkin et al. 2006)

probabilistic pairwise Markov model (PPMMs) for detecting prostate cancer in whole-mount histological images. The algorithm was integrated into a Computer Aided Diagnosis system which aims to detect cancerous glands. Experiments were performed on a dataset which consisted of 20 histological sections of prostate tissue taken from 19 patients. Their evaluations revealed an Area Under the Curve of $87 \%$ when the probabilistic pairwise Markov model (PPMMs) was used, as opposed to the alternative baseline system which returned an Area Under the Curve of 83\%. Yu et al. (2011) applied the Probabilistic Pairwise Markov model for detecting regions of prostate cancer using colour fractal dimensions. Their approach combined the probability map constructed via colour fractal dimension with their Probabilistic Pairwise Markov model (PPMM). The performance of the detection system was evaluated using 27 prostatectomy specimens from 10 patients. The results revealed an Area Under the Curve of 83.1\%. Markov models are useful to adopt when a clinical decision problem involves risk that is continuous over time, when the timing of events is important, and when 
jimportant events may occur more than once (such as cancer recurrence) (Sonnenberg \& Beck, 1993). Markov models are recursive decision trees with the advantage over the conventional decision trees that they can model situations under uncertainty which consider timing of events which are too complex for conventional decision trees to model. With regards to Markov models that have been developed to solve clinical decision problems (such as life expectancy estimation, and decision for screening), the Markov-cycle tree is a suitable model. The Markov-cycle tree uses a tree representation of clinical events and may be evaluated either as a cohort simulation or as a Monte Carlo simulation (Sonnenberg \& Beck, 1993). Fig. 9 shows an example of a Markov cycle tree for the analysis of lung cancer screening that includes four health states: well, asymptomatic lung cancer (ASYM), symptomatic lung cancer (SYM), and dead. The decision making process starts at time zero, when the cohort of patients subject to screening are distributed between the 'well' and 'ASYM' states (Black, 1999). The cohort of patients then moves between the asymptomatic, symptomatic and dead (from cancer, or other causes) states. Given that each living health state (well, asymptomatic and symptomatic) is assigned a value of 1 , and the dead state is assigned a value of 0 , then the Markov model can be used to estimate life expectancy (Black, 1999).

Markov models have also been applied for finding optimal prostate cancer treatment plans. Goulionis \& Koutsiumaris (2010) developed a Markov-based model (namely the Partially observable Markov decision model) for finding optimal disease treatment plans. Smith et al. (2009) created a Markov model as an aid for selecting the radiation-therapy strategy for patients with prostate cancer. Ross et al. (2005) created a Markov model to estimate the benefits of screening for prostate cancer as patient age at screening increases. Recently, Sanyal et al. (2014) developed a Markov Monte Carlo model for predicting clinical outcomes and mortality across risk groups, with the ultimate aim of better managing disease. In order to determine the cost-effectiveness of prostate cancer treatment, Keegan et al. (2012) and Eldefrawy et al. (2013) applied the Markov model for determining an economic analysis of active surveillance compared with imme- 


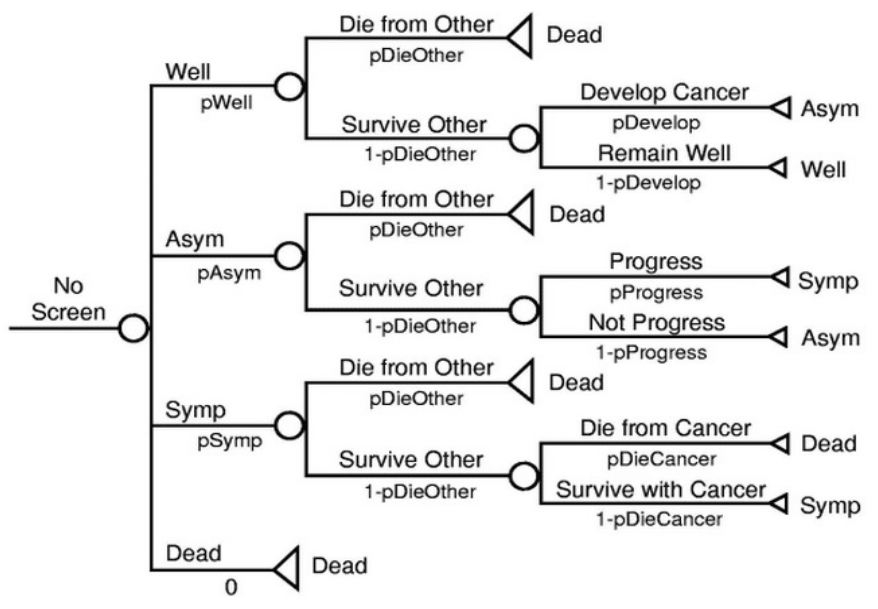

Figure 9: Markov cycle tree for natural history of disease. Asym = asymptomatic; Symp = symptomatic. Black (1999)

diate treatment. Black (1999) emphasise that although Randomised Controlled Trials (RCTs) are the most valid method for determining the effectiveness of screening, they require a large number of participants and many years of followup. Decision models, such as the Markov model, can be effectively used to find answers to clinical problems, and they can help to address, in a timely manner, many of the important clinical questions about cancer screening which have not been answered by randomised controlled trials. Markov models can be used to predict many outcomes related to screening and the expected gain (or loss) in life expectancy with screening. Sanyal et al. (2014) also point out the limitations of randomised controlled trials and argue that "most of the observational studies and randomized trials on prostate cancer have concurrently evaluated fewer treatments over short follow-up" and that decision analytic models can be used to address the lack of literature on evaluations of contemporary management options.

There is a need to apply decision making models such as the Markov model for forecasting short and long-term clinical outcomes of evaluating various contemporary management options. 


\section{Selecting a suitable Metaheuristic Optimisation method}

Many studies have focused on the topic of comparing the performance and

1090 Kachitvichyanukul, 2012), (Xue et al. 2016), (Hassan et al., 2005), (Elbeltagi et al. 2005). The task of comparing optimisation methods is itself a challenge, since although the objective functions and the fitness values of their solutions could be the same, the optimisation methods may select different individual

features to reach that best solution. For this reason it is important to experiment with various methods and consider the difference of the fitness values; the consistency of the selected features during the comparison process; and the time taken for the method to reach the best solution (Xue et al., 2016).

Elbeltagi et al. (2005) evaluated the performance of various optimisation algorithms including the GA, the PSO, and ACO using benchmark problems. Twenty trial runs were performed for each problem, and the performance of the different algorithms was compared using three evaluation criteria: (1) the percentage of success, as represented by the number of trials required for the objective function to reach its known target value; $(2)$ the average value of the solution obtained in all trials; and (3) the processing time to reach the optimum target value. It was observed that the performance of the algorithms depended on the objective function which the algorithm was trying to maximise or minimise. Experimenting with various objective functions, the authors concluded that the behaviour of each optimisation algorithm in all continuous and discrete test problems was consistent. They found that the PSO algorithm outperformed all other algorithms in terms of solution accuracy and success rate, and was second best in terms of processing time (Elbeltagi et al. 2005). The computational effort required by PSO to arrive to optimum solutions is less than the effort required to arrive at the same solutions by the GA, due to its simple updating mechanisms - the PSO generates a new swarm of particles via the velocity and position update equations. 
Hassan et al. (2005) compared the PSO with the GA and in particular they carried out two tests (1) an effectiveness test to measure the quality of the solutions found by the two algorithms with respect to known solutions for benchmark test problems; and (2) an efficiency test, to determine whether the computational effort of PSO is less than that of the GA for the sample problem set using the same convergence criteria. Their results revealed that the computational effort required by PSO to arrive to high quality solutions is less than the effort required to arrive at the same high quality solutions by the GA. Importantly, it was concluded that the difference in computational effort between PSO and the GA is problem dependent. It appears that PSO outperforms the GA when applied to solve unconstrained nonlinear problems rather than constrained nonlinear problems using continuous or discrete design variables.

Certainly, the first consideration when choosing which metaheuristic method to use is the type of optimisation problem, and in particular whether it is a continuous optimisation or a discrete optimisation problem. Some methods work best with discrete variables, often a subset of integers, whereas other methods can work with variables that can take on any real value. Optimisation methods with discrete variables are known as discrete optimisation problems; whereas methods with continuous variables are continuous optimisation problems.

GA is initially a discrete optimisation technique that is also suitable for combinatorial optimisation problems, whereas PSO is a continuous technique that is very poorly suited to combinatorial problems. Discrete or combinatorial optimisation deals mainly with problems where the optimum solution must be found from a finite number of possible solutions. For this reason the GA and ACO algorithm are both suited to discrete problems. However, metaheuristic algorithms which were originally developed to solve continuous optimisation problems, such as PSO, have been adapted to solve discrete optimisation problems. For example, Yang et al. (2014) proposed a new multi-objective discrete PSO algorithm based on enhanced search strategy.

Most metaheuristic optimisation methods (including GA, PSO, and ACO) have been applied for feature selection or to optimise the performance of classi- 
fiers using datasets containing thousands of features. Of all metaheuristic optimisation methods, the GA is the most established algorithm with most versions

Metaheuristic optimisation algorithms, such as the GA, PSO, ACO, and the AIS are stochastic search methods, meaning that they have randomness in their nature, and will therefore usually reach a different solution every time they are executed. In particular, stochastic optimisation is the process of maximizing or minimizing the value of an objective function when one or more of the input parameters is subject to randomness. This can pose a problem when datasets have a large number of features and thus optimisation algorithms whose search strategies can converge to a solution in a reasonable amount of time and consistently need to be chosen. Xue et al. (2016) emphasize that for large-scale optimisation problems, and especially feature selection problems, new approaches are needed, including new search algorithms. Metaheuristic optimisation approaches have shown their potential for large-scale (global) optimisation problems and which provides a good opportunity to better address large-scale feature selection tasks.

In conclusion, it is important to adopt several suitable optimisation methods and evaluate these using the dataset, objective functions, and suitable evaluation strategies to determine most suitable method for the task at hand. Certainly, when choosing an algorithm it is worth experimenting with the Genetic algorithm for discrete combinatorial optimisation problems and the PSO for continuous optimisation problems.

\section{Discussion and Future Directions}

Computational intelligence based models are suitable for clinical and biological predictions as they are efficient in finding patterns in data which contain noisy features, and which traditional statistical models alone often fail to handle.

A commonly encountered challenge in cancer research is the collection of a representative data sample of sufficient size to enable the algorithms to learn on a subset of that data, and to predict on an unseen subset of data. This problem 
is more apparent in research concerning image analysis. It has been observed that many studies do not place appropriate emphasis on adopting a suitable validation approach for evaluating the performance of algorithms. The dataset size, dataset quality, and quality of selected features impact on the effectiveness of computational intelligence approaches for accurate cancer predictions. In general, computational intelligence approaches perform well on cancer risk prediction tasks and, due to their capability to be retrained on new data and to adapt their algorithm in order to further improve prediction, these approaches are much more efficient for risk prediction than statistical approaches. Suitable validation techniques (i.e. k-fold, leave-one-out, etc.) must be carefully considered and adopted for evaluating system performance appropriately in order to accurately report results. Computational intelligence algorithms which are over trained (and hence are susceptible to overfitting of the data) are likely to report excellent training and poor testing and validation results. It is therefore important to adopt a suitable cross validation approach. The remaining subsections summarise the advances and challenges of computational intelligence approaches, as applied to the analysis and interpretation of cancer data.

\subsection{Predictive Modeling using Multimodal Data (Image and Non-Image)}

Data obtained from various sources such as patient examinations, blood tests and images can hold important information which is needed in order to reach a more accurate cancer prediction. However, not all data features are needed and it is important to select the best features (i.e. predictors) from each set of data. The feature selection step is important because unnecessary features will add noise and obscure a predictive model's performance. Importantly, due to the uncertainty and noise found in the different types of data, it is useful and important to take into consideration multimodal data when making predictions which can affect decision-making. Selecting the best features from the different types of data and finding the best combinations for solving the prediction task is a significant challenge. Liang et al. (2015) emphasise that "Although numerous integrative clustering approaches have been developed to analyse cancer data, 
few of them are particularly designed to exploit both deep intrinsic statistical properties of each input modality and complex cross-modality correlations among multi-platform input data". Although the solution proposed by Liang et al. (2015) is not specific to prostate cancer, it is worth mentioning. They propose a multimodal Deep Belief Network (DBN) approach which initially encodes the relationships of features found in each data modality, into multiple layers of hidden variables, and then uses a joint latent model to fuse common features derived from multiple input modalities. The Contrastive Divergence (CD) learning algorithm is then applied to infer the parameters of a multimodal Deep Belief network model in an unsupervised manner. More research which demonstrates the benefits of multimodal data in improving cancer predictions needs to be performed. Due to the uncertainty and noise found in data obtained from images, and the challenges involved in identifying the cancerous regions with confidence, combining image with non-image data is important for improving predictions.

For complex problems, an Ensemble of learning algorithms can be used to find a solution when single classification algorithms are not capable of reaching sufficient accuracy, and the results of a number of classifiers needs to be considered as part of reaching a predicted outcome. The suitability of Ensemble Learning approaches for multimodal data needs to be thoroughly investigated.

\subsubsection{Big Data in Prostate Radiotherapy}

Big data applied to radiation oncology involves the use of multimodal data which includes clinical features, treatment related dose-volume metrics and biological data. Given the heterogeneity of patient populations, it is important that datasets are large enough to include enough records to reflect the underlying patient population (Coates et al., 2016). Coates et al. Coates et al. (2016) provide a review of big data-mining techniques that have been applied to radiotherapy outcome modeling. They found that a major limitation in modern outcome modeling is the difficulty in grouping together datasets from multiple institutions, mainly due to patient privacy and security concerns. They pro- 
pose that appropriate data-sharing protocols are needed in order to enable big data analytics to be applied. This will result in improved data-driven outcome models, as these will be trained and validated on larger datasets.

\subsubsection{Micro-array gene expression analysis}

A number of studies are focusing on micro-array gene expression data for cancer diagnosis. Although these studies mainly utilise statistical approaches for identifying and classifying biomarkers, computational intelligence approaches could also be applied to improve the accuracy of these statistical models. According to Alexey et al. (2015) "a significant need for reliable and accurate cancer diagnostics and prognosis compels the search for novel biomarkers that would be able to discriminate between indolent and aggressive tumours at the early stages of disease." Very few studies on the use of machine learning and computational intelligence approaches for developing predictive prostate cancer models using RNA-Sequencing exist(Singireddy et al., 2015). RNA-sequencing (Wang et al., 2009) is an approach that is rapidly revealing promising results in prostate cancer. For example, a study by Shi et al. (2015) proposed using miR-124, a small non-coding RNA, in patients to make enzalutamide therapy more effective. Enzalutamide is a new type of hormone therapy for men whose prostate cancer has spread to other parts of the body (advanced stage of prostate cancer) and has stopped responding to other hormone therapy treatments. Enzalutamide therapy may help some men to live longer and can also help to control symptoms.

\subsection{Feature Selection}

Feature selection is the process of finding the best predictor variables which can be used for maximising predictive accuracy. Although these variables can be identified using statistical tests, these approaches may not be suitable for high dimensional data, in that statistical algorithms can return a high number of false positives due to random correlations (Handl et al., 2007). Extracting relevant features from microarray data is a challenging task, as these data comprise a 
large number of features with few samples being generally available (LuqueBaena et al. 2014). There is a need for the development of suitable feature identification approaches which can be applied to biomarker data.

Computational intelligence approaches, and in particular, metaheuristic approaches can be effective and efficient solutions to feature selection. Such approaches may be particularly useful when dealing with a large number of variables and there is a requirement to select the most suitable ones for optimising the prediction task. In particular, feature selection approaches which are based on metaheuristic optimisation algorithms are capable of finding the best combination of features. These features are the predictors which would be used to train a classifier. Statistical approaches can be embedded as objective functions in metaheuristic optimisation approaches.

Only a few prostate cancer studies have applied the Genetic Algorithm and other metaheuristic approaches to prostate cancer prediction tasks. More studies are needed for investigating the performance of computational intelligence algorithms applied to multimodal data; determining which approaches are most efficient for feature selection and extraction in multimodal and non-multimodal data; and whether the performance of prediction models can be improved when such approaches are adopted.

\subsection{Understanding the Reasoning Behind Predictions}

Artificial Neural Networks, Support Vector Machines, and Naive Bayesian are among the most popular approaches that have been used for predicting prostate cancer risk and are known to perform well on prediction tasks. However, it is impossible for the human to understand the reasoning behind the predictions derived from the above-mentioned approaches. In contrast, Fuzzy rule-based approaches and Bayesian networks have the additional benefit of presenting the reasoning behind a prediction (either in the form of rules, or a graphical representation of variable associations) which can be easily interpreted by humans, and therefore can provide a qualitative reasoning to the risk prediction process. Although Bayesian networks are amongst the most useful 
approaches for presenting reasoning behind a prediction, the most challenging task in dealing with Bayesian networks is learning their structure. Heuristic search algorithms such as the hill-climbing search heuristic approach (Tsamardinos et al., 2006), and Genetic Algorithms (Larranaga et al., 1997) therefore need

\section{Conclusion}

Computational intelligence approaches have not been as widely adopted into prostate cancer detection as they have for other diseases and it is now time to embrace, challenge and expose these approaches to achieve better results in prostate cancer predictive modeling tasks. In the future, clinical data could

${ }_{1320}$ be combined with novel biomarkers and imaging tools to improve accuracy in risk prediction, prognosis and diagnosis tasks. Advanced feature selection approaches can be applied to find the most significant features from a variety of data sources and bring these together for more accurate prostate cancer prediction models. Despite the evidence in the literature that computational intel- 
Midlands. The authors would also like to acknowledge the financial support of The Leverhulme Trust (Research Project Grant RPG-2016-252).

\section{References}

Alexey, A. D., Eugenia, E., Rosenberg, G. S., \& Krasnov, e. a. (2015). Identification of novel epigenetic markers of prostate cancer by noti-microarray analysis. Disease Markers, 2015.

Arevalo, J. E., Cruz-Roa, A., Arias, V., Romero, E., \& González, F. A. (2015a). An unsupervised feature learning framework for basal cell carcinoma image analysis. Artificial Intelligence in Medicine, 64, 131-145.

1350

Arevalo, J. E., González, F. A., Ramos-Pollán, R., Oliveira, J. L., \& GuevaraLópez, M. Á. (2015b). Convolutional neural networks for mammography mass lesion classification. In $E M B C$ (pp. 797-800). IEEE. 
Azizi, S., Imani, F., Zhuang, B., Tahmasebi, A., Kwak, J. T., Xu, S., Uniyal, N., Turkbey, B., Choyke, P., Pinto, P., Wood, B., Moradi, M., Mousavi, P., \& Abolmaesumi, P. (2015). Medical image computing and computerassisted intervention - miccai 2015: 18th international conference, munich, germany, october 5-9, 2015, proceedings, part ii. chapter Ultrasound-Based Detection of Prostate Cancer Using Automatic Feature Selection with Deep Belief Networks. (pp. 70-77). Cham: Springer International Publishing.

Balachandran, K., \& Anitha, R. (2013). Ensemble based optimal classification model for pre-diagnosis of lung cancer. In Computing, Communications and Networking Technologies (ICCCNT), 2013 Fourth International Conference on (pp. 1-7). IEEE.

Benecchi, L. (2006). Neuro-fuzzy system for prostate cancer diagnosis. Urology, $68,357-361$.

Bengio, Y. (2013). Statistical language and speech processing: First international conference, slsp 2013, tarragona, spain, july 29-31, 2013. proceedings. In A.-H. Dediu, C. Martín-Vide, R. Mitkov, \& B. Truthe (Eds.), Deep Learning of Representations: Looking Forward (pp. 1-37). Berlin, Heidelberg: Springer Berlin Heidelberg.

Bianchi, L., Dorigo, M., Gambardella, L., \& Gutjahr, W. (2009). A survey on metaheuristics for stochastic combinatorial optimization. Natural Computing, $8,239-287$.

Black, W. C. (1999). Should this patient be screened for cancer? Effective Clinical Practice, 2, 86-95.

Bourdes, V., Bonnevay, S., Lisboa, P. J., Aung, M. H., Chabaud, S., Bachelot, T., Perol, D., \& Négrier, S. (2007). Breast cancer predictions by neural networks analysis: a comparison with logistic regression. In Engineering in Medicine and Biology Society, 200\%. EMBS 200\%. 29th Annual International Conference of the IEEE (pp. 5424-5427). IEEE. 
Breiman, L. (2001). Random forests. Mach. Learn., 45, 5-32.

Castanho, M., Hernandes, F., De Ré, A., Rautenberg, S., \& Billis, A. (2013).

Fuzzy expert system for predicting pathological stage of prostate cancer. Expert Systems with Applications, 40, 466-470.

1385 Çinar, M., Engin, M., Engin, E. Z., \& Ziya Ateşçi, Y. (2009). Early prostate cancer diagnosis by using artificial neural networks and support vector machines. Expert Syst. Appl., 36, 6357-6361.

Chadha, K., Miller, A., Nair, B., Schwartz, S., Trump, D., \& Underwood, W. (2014). New serum biomarkers for prostate cancer diagnosis. Clinical Cancer Investigation Journal, 3, 72-79.

Choukroun, D., Bar-Itzhack, I. Y., \& Oshman, Y. (2006). Novel quaternion kalman filter. IEEE Transactions on Aerospace and Electronic Systems, 42, 174-190.

Ciresan, D., Meier, U., \& Schmidhuber, J. (2012). Multi-column deep neural networks for image classification. In Proceedings of the 2012 IEEE Conference on Computer Vision and Pattern Recognition (CVPR) CVPR '12 (pp. 36423649). Washington, DC, USA: IEEE Computer Society.

Coates, J., Souhami, L., \& El Naqa, I. (2016). Big data analytics for prostate radiotherapy. Frontiers in Oncology, 6, 149.

Cortes, C., \& Vapnik, V. (1995). Support-vector networks. Mach. Learn., 20, 273-297.

Cosma, G., Acampora, G., Brown, D., Rees, R. C., Khan, M., \& Pockley, A. G. (2016). Prediction of pathological stage in patients with prostate cancer: A neuro-fuzzy model. PLoS ONE, 11, 1-27.

Dass, M. V., Rasheed, M. A., \& Ali, M. M. (2014). Classification of lung cancer subtypes by data mining technique. In Control, Instrumentation, Energy and 
Communication (CIEC), 2014 International Conference on (pp. 558-562).

IEEE.

Diaz, J. M., Pinon, R. C., \& Solano, G. (2014). Lung cancer classification using genetic algorithm to optimize prediction models. In Information, Intelligence, Systems and Applications, IISA 2014, The 5th International Conference on (pp. 1-6). IEEE.

Dice, L. R. (1945). Measures of the amount of ecologic association between species. Ecology, 26, 297-302.

Djavan, B., Remzi, M., Zlotta, A., Seitz, C., Snow, P., \& Marberger, M. (2002). Novel artificial neural network for early detection of prostate cancer. Journal of Clinical Oncology, 20, 921-929.

Dorigo, M., \& Gambardella, L. M. (1997). Ant colony system: A cooperative learning approach to the traveling salesman problem. Trans. Evol. Comp, 1, $53-66$.

Doyle, S., Feldman, M., Tomaszewski, J., \& Madabhushi, A. (2012). A boosted bayesian multiresolution classifier for prostate cancer detection from digitized needle biopsies. Biomedical Engineering, IEEE Transactions on, 59, 12051218. Miller, K., \& Stephan, C. (2012). External validation of an artificial neural network and two nomograms for prostate cancer detection. ISRN Urology, 2012.

Edge, S., Byrd, D., Compton, C., Fritz, A., Greene, F., \& Trotti, A. (2010). AJCC Cancer Staging Manual. (7th ed.). New York, NY: Springer.

Elbeltagi, E., Hegazy, T., \& Grierson, D. (2005). Comparison among five evolutionary-based optimization algorithms. Adv. Eng. Inform., 19, 43-53. 
Eldefrawy, A., Katkoori, D., Abramowitz, M., Soloway, M. S., \& Manoharan, M. (2013). Active surveillance vs. treatment for low-risk prostate cancer: A

Froelich, W., Papageorgiou, E. I., Samarinas, M., \& Skriapas, K. (2012). Application of evolutionary fuzzy cognitive maps to the long-term prediction of prostate cancer. Applied Soft Computing, 12, 3810-3817.

Gaul, D., Mezencev, R., Long, T., Jones, C., Benigno, B., Gray, A., Fernández, 1455 F., \& McDonald, J. (2015). Highly-accurate metabolomic detection of earlystage ovarian cancer. Scientific Reports, 5 .

Gertych, A., Ing, N., Ma, Z., Fuchs, T. J., Salman, S., Mohanty, S., Bhele, S., Velsquez-Vacca, A., Amin, M. B., \& Knudsen, B. S. (2015). Machine learning approaches to analyze histological images of tissues from radical prostatec- 
tomies. Computerized Medical Imaging and Graphics, 46, Part 2, $197-208$. Information Technologies in Biomedicine.

Ghahramani, Z. (2002). Hidden markov models. In An Introduction to Hidden Markov Models and Bayesian Networks (pp. 9-42). River Edge, NJ, USA: World Scientific Publishing Co., Inc.

Golugula, A., Lee, G., Master, S. R., Feldman, M. D., Tomaszewski, J. E., Speicher, D. W., \& Madabhushi, A. (2011). Supervised regularized canonical correlation analysis: Integrating histologic and proteomic measurements for predicting biochemical recurrence following prostate surgery. BMC bioinformatics, 12, 483.

Goulionis, J. E., \& Koutsiumaris, B. K. (2010). Partially observable markov decision model for the treatment of early prostate cancer. Opsearch, 47, $105-117$.

Greenblatt, A., Mosquera-Lopez, C., \& Agaian, S. (2013). Quaternion neural networks applied to prostate cancer gleason grading. In Systems, Man, and Cybernetics (SMC), 2013 IEEE International Conference on (pp. 1144-1149).

Guo, Y., Gao, Y., \& Shen, D. (2015). Deformable MR prostate segmentation via deep feature learning and sparse patch matching. IEEE Transactions on Medical Imaging, PP, 1-1.

Guo, Y., Liu, Y., Oerlemans, A., Lao, S., Wu, S., \& Lew, M. S. (2016). Deep learning for visual understanding: A review. Neurocomputing, 187, $27-48$. Recent Developments on Deep Big Vision.

Hameed, W. A., \& Bagavandas, M. (2011). Comparison of fuzzy and neural network models to diagnose breast cancer. In Control, Computation and Information Systems (pp. 241-248). Springer.

Han, J., Kamber, M., \& Pei, J. (2011). Data Mining: Concepts and Techniques. (3rd ed.). San Francisco, CA, USA: Morgan Kaufmann Publishers Inc. 
Handl, J., Kell, D. B., \& Knowles, J. (2007). Multiobjective optimization in bioinformatics and computational biology. IEEE/ACM Trans. Comput. Biol. Bioinformatics, 4, 279-292.

Haq, N. F., Kozlowski, P., Jones, E. C., Chang, S. D., Goldenberg, S. L., \& Moradi, M. (2015). A data-driven approach to prostate cancer detection from dynamic contrast enhanced MRI. Computerized Medical Imaging and Graphics, 41, 37-45.

Hassan, R., Cohanim, B., De Weck, O., \& Venter, G. (2005). A comparison of particle swarm optimization and the genetic algorithm. In Proceedings of the 1st AIAA multidisciplinary design optimization specialist conference (pp. $18-21)$.

Hastie, T., Tibshirani, R., \& Friedman, J. (2001). The Elements of Statistical Learning. Springer Series in Statistics. New York, NY, USA: Springer New York Inc.

Hinton, G. E., \& Sejnowski, T. J. (1986). Learning and relearning in boltzmann machines. Parallel Distributed Processing: Explorations in the Microstructure of Cognition, 1, 282-317.

Holland, J. H. (1992). Adaptation in Natural and Artificial Systems: An Introductory Analysis with Applications to Biology, Control and Artificial Intelligence. Cambridge, MA, USA: MIT Press.

Jerne, N. K. (1974). Towards a network theory of the immune system. Annales d'immunologie, 125C, 373-389.

Jiang, X., Xue, D., Brufsky, A., Khan, S., \& Neapolitan, R. (2014). A new method for predicting patient survivorship using efficient Bayesian Network Learning. Cancer Informatics, 13, 47-57.

Kachitvichyanukul, V. (2012). Comparison of three evolutionary algorithms: Ga, pso, and de. Industrial Engineering and Management Systems, 11, 215223. 
1515 Karakiewicz, P. I., Benayoun, S., Kattan, M. W., Perrotte, P., Valiquette, L., Scardino, P. T., Cagiannos, I., Heinzer, H., Tanguay, S., Aprikian, A. G. et al. (2005). Development and validation of a nomogram predicting the outcome of prostate biopsy based on patient age, digital rectal examination and serum prostate specific antigen. The Journal of Urology, 173, 1930-1934.

1520 Kawakami, S., Numao, N., Okubo, Y., Koga, F., Yamamoto, S., Saito, K., Fujii, Y., Yonese, J., Masuda, H., Kihara, K. et al. (2008). Development, validation, and head-to-head comparison of logistic regression-based nomograms and artificial neural network models predicting prostate cancer on initial extended biopsy. European Urology, 54, 601-611.

${ }_{1525}$ Keegan, K. A., A, D. M., Durbin-Johnson, B., \& Evans, C. P. (2012). Active surveillance for prostate cancer compared with immediate treatment. Cancer, $118,3512-3518$.

Keles, A., Hasiloglu, A. S., Keles, A., \& Aksoy, Y. (2007). Neuro-fuzzy classification of prostate cancer using NEFCLASS-J. Computers in Biology and $1530 \quad$ Medicine, 37, $1617-1628$.

Kennedy, J., \& Eberhart, R. (1995). Particle swarm optimization. In Neural Networks, 1995. Proceedings., IEEE International Conference on (pp. 19421948 vol.4). volume 4 .

Kim, S. G., \& Seo, Y. G. (2013). A TRUS prostate segmentation using gabor texture features and snake-like contour. Journal of Information Processing Systems, 9, 103-116.

Kohavi, R. (1995). A study of cross-validation and bootstrap for accuracy estimation and model selection. In Proceedings of the 14 th International Joint Conference on Artificial Intelligence - Volume 2 IJCAI'95 (pp. 1137-1143). San Francisco, CA, USA: Morgan Kaufmann Publishers Inc.

Kosko, B. (1986). Fuzzy cognitive maps. International Journal of Man-Machine Studies, 24, $65-75$. 
Kumar, S. A., Ramesh, J., Vanathi, P., \& Gunavathi, K. (2011). Robust and automated lung nodule diagnosis from CT images based on fuzzy systems. In Process Automation, Control and Computing (PACC), 2011 International Conference On (pp. 1-6). IEEE.

Kuo, R., Chen, S. S., Cheng, W. C., \& Tsai, C. (2014). Integration of artificial immune network and k-means for cluster analysis. Knowl. Inf. Syst., 40, $541-557$.

Kuo, R.-J., Huang, M.-H., Cheng, W.-C., Lin, C.-C., \& Wu, Y.-H. (2015). Application of a two-stage fuzzy neural network to a prostate cancer prognosis system. Artificial intelligence in medicine, 63, 119-133.

Lacave, C., \& Dez, F. J. (2003). Knowledge acquisition in PROSTANET a Bayesian network for diagnosing prostate cancer. In V. Palade, R. J. Howlett, \& L. Jain (Eds.), Knowledge-Based Intelligent Information and Engineering Systems (pp. 1345-1350). Springer Berlin Heidelberg volume 2774 of Lecture Notes in Computer Science.

Larranaga, P., Sierra, B., Gallego, M. J., Michelena, M. J., \& Picaza, J. M. (1997). Learning Bayesian Networks by Genetic Algorithms: A case study in the prediction of survival in malignant skin melanoma. In E. Keravnou, C. Garbay, R. Baud, \& J. Wyatt (Eds.), Artificial Intelligence in Medicine (pp. 261-272). Springer Berlin Heidelberg volume 1211 of Lecture Notes in Computer Science.

LeCun, Y., Bengio, Y., \& Hinton, G. (2015). Deep learning. Nature, 521, 1565 436444.

Lee, H., Grosse, R., Ranganath, R., \& Ng, A. Y. (2009). Convolutional deep belief networks for scalable unsupervised learning of hierarchical representations. In Proceedings of the 26th Annual International Conference on Machine Learning ICML '09 (pp. 609-616). New York, NY, USA: ACM. 
1570 Lee, H. J., Kim, K. G., Lee, S. E., Byun, S.-S., Hwang, S. I., Jung, S. I., Hong, S. K., \& Kim, S. H. (2006). Role of transrectal ultrasonography in the prediction of prostate cancer artificial neural network analysis. Journal of Ultrasound in Medicine, 25, 815-821.

Lehaire, J., Flamary, R., Rouviere, O., \& Lartizien, C. (2014). Computer-aided diagnostic system for prostate cancer detection and characterization combining learned dictionaries and supervised classification. In Image Processing (ICIP), 2014 IEEE International Conference on (pp. 2251-2255). IEEE.

Liang, M., Li, Z., Chen, T., \& Zeng, J. (2015). Integrative data analysis of multiplatform cancer data with a multimodal deep learning approach. IEEE/ACM Transactions on Computational Biology and Bioinformatics, 12, 928-937.

Luque-Baena, R. M., Urda, D., Subirats, J. L., Franco, L., \& Jerez, J. M. (2014). Application of genetic algorithms and constructive neural networks for the analysis of microarray cancer data. Theoretical Biology and Medical Modelling, 11, 1-18.

1585 Martens, D., De Backer, M., Haesen, R., Vanthienen, J., Snoeck, M., \& Baesens, B. (2007). Classification with ant colony optimization. Evolutionary Computation, IEEE Transactions on, 11, 651-665.

Matulewicz, L., Jansen, J. F., Bokacheva, L., Vargas, H. A., Akin, O., Fine, S. W., Shukla-Dave, A., Eastham, J. A., Hricak, H., Koutcher, J. A. et al. (2014). Anatomic segmentation improves prostate cancer detection with artificial neural networks analysis of $1 \mathrm{H}$ magnetic resonance spectroscopic imaging. Journal of Magnetic Resonance Imaging, 40, 1414-1421.

Mazzetti, S., De Luca, M., Bracco, C., Vignati, A., Giannini, V., Stasi, M., Russo, F., Armando, E., Agliozzo, S., \& Regge, D. (2011). A CAD system based on multi-parametric analysis for cancer prostate detection on DCEMRI. In SPIE Medical Imaging (pp. 79633Q-79633Q). International Society for Optics and Photonics. 
McGeachy, P., Madamesila, J., Beauchamp, A., \& Khan, R. (2015). An opensource genetic algorithm for determining optimal seed distributions for lowdose-rate prostate brachytherapy. Brachytherapy, 14, 692-702.

Mikolov, T., Sutskever, I., Chen, K., Corrado, G. S., \& Dean, J. (2013). Distributed representations of words and phrases and their compositionality. Advances in Neural Information Processing Systems 26, (pp. 3111-3119).

Mohareri, O., Ruszkowski, A., Lobo, J., Ischia, J., Baghani, A., Nir, G., Eskandari, H., Jones, E., Fazli, L., Goldenberg, L. et al. (2014). Multi-parametric 3D quantitative ultrasound vibro-lastography imaging for detecting palpable prostate tumors. In Medical Image Computing and Computer-Assisted Intervention-MICCAI 2014 (pp. 561-568). Springer.

Monaco, J., Tomaszewski, J. E., Feldman, M. D., Moradi, M., Mousavi, P., 1610 Boag, A., Davidson, C., Abolmaesumi, P., \& Madabhushi, A. (2009). Probabilistic pairwise Markov models: application to prostate cancer detection. Proc. SPIE, 7259, 725903-725903-12.

Mumford, C. L., \& Jain, L. C. (2009). Computational Intelligence: Collaboration, Fusion and Emergence. (1st ed.). Springer Publishing Company, Incorporated.

Ni, Y., Stingo, F. C., \& Baladandayuthapani, V. (2014). Integrative Bayesian Network Analysis of genomic data. Cancer Informatics, (pp. 39-48).

Ospina, J., Zhu, J., Chira, C., Bossi, A., Delobel, J., Beckendorf, V., B, D., Lagrange, J., Correa, J., Simon, A., Acosta, O., \& Crevoisier, R. (2014). Random forests to predict rectal toxicity following prostate cancer radiation therapy. International Journal of Radiation Oncology, 89, 1024 - 1031.

Parpinelli, R., Lopes, H., \& Freitas, A. (2002). Data mining with an ant colony optimization algorithm. Evolutionary Computation, IEEE Transactions on, $6,321-332$. 
Peng, H., Long, F., \& Ding, C. (2005). Feature selection based on mutual information: Criteria of max-dependency, max-relevance, and min-redundancy. IEEE Trans. Pattern Anal. Mach. Intell., 27, 1226-1238.

Pieczynski, W. (2003). Pairwise Markov chains. IEEE Transactions on Pattern Analysis and Machine Intelligence, 25, 634-639.

Polikar, R. (2009). Ensemble learning. Scholarpedia, 4, 2776.

Ross, K. S., Guess, H. A., \& Carter, H. B. (2005). Estimation of treatment benefits when PSA screening for prostate cancer is discontinued at different ages. Urology, 66, 1038-1042.

Russell, S., Binder, J., Koller, D., \& Kanazawa, K. (1995). Local learning in probabilistic networks with hidden variables. In Proceedings of the 14th International Joint Conference on Artificial Intelligence - Volume 2 IJCAI'95 (pp. 1146-1152). San Francisco, CA, USA: Morgan Kaufmann Publishers Inc.

Sadoughi, F., \& Ghaderzadeh, M. (2013). A hybrid particle swarm and neural network approach for detection of prostate cancer from benign hyperplasia of prostate. Studies in Health Technology and Informatics, 205, 481-485.

Salakhutdinov, R., \& Hinton, G. E. (2009). Deep boltzmann machines. In Proceedings of the Twelfth International Conference on Artificial Intelligence and Statistics, AISTATS 2009, Clearwater Beach, Florida, USA, April 16-18, 2009 (pp. 448-455).

1645 Sanyal, C., Aprikian, A., Cury, F., Chevalier, S., \& Dragomir, A. (2014). Clinical management and burden of prostate cancer: A markov monte carlo model. PLoS ONE, 9, e113432.

Saritas, I., Ozkan, I. A., \& Sert, I. U. (2010). Prognosis of prostate cancer by artificial neural networks. Expert Systems with Applications, 37, 6646-6650.

1650 Shariat, S., Kattan, M., Vickers, A., Karakiewicz, P., \& P.T., S. (2009a). Critical review of prostate cancer predictive tools. Future Oncology, 510, 1555-1584. 
Shariat, S. F., Kattan, M. W., Vickers, A. J., Karakiewicz, P. I., \& Scardino, P. T. (2009b). Critical review of prostate cancer predictive tools. Future Oncol, 5, 1555-84.

Stephan, C., Cammann, H., Deger, S., Schrader, M., Meyer, H. A., Miller, K., Lein, M., \& Jung, K. (2009). Benign prostatic hyperplasia-associated free prostate-specific antigen improves detection of prostate cancer in an artificial neural network. Urology, 74, $873-877$.

Strobl, A. N., Vickers, A. J., Calster, B. V., Steyerberg, E., Leach, R. J., Thompson, I. M., \& Ankerst, D. P. (2015). Improving patient prostate cancer risk 
assessment: Moving from static, globally-applied to dynamic, practice-specific risk calculators. Journal of Biomedical Informatics, 56, 87-93.

Suk, H.-I., Lee, S.-W., \& Shen, D. (2014). Hierarchical feature representation and multimodal fusion with deep learning for AD/MCI diagnosis. NeuroImage, $101,569-582$.

Sun, A., Tan, Y., \& Zhang, D. (2008). Hybrid prediction model based on BP neural network for lung cancer. In IT in Medicine and Education, 2008. ITME 2008. IEEE International Symposium on (pp. 532-535). IEEE.

Sung, Y. S., Kwon, H.-J., Park, B.-W., Cho, G., Lee, C. K., Cho, K.-S., \& Kim, J. K. (2011). Prostate cancer detection on dynamic contrast-enhanced MRI: Computer-aided diagnosis versus single perfusion parameter maps. American Journal of Roentgenology, 197, 1122-1129.

Swan, A. L., Mobasheri, A., Allaway, D., Liddell, S., \& Bacardit, J. (2013). Application of machine learning to proteomics data: Classification and biomarker identification in postgenomics biology. OMICS: A Journal of Integrative Biology, 17, 595610.

Tang, N., \& Vemuri, V. R. (2005). An artificial immune system approach to document clustering. In H. Haddad, L. M. Liebrock, A. Omicini, \& R. L. Wainwright (Eds.), SAC (pp. 918-922). ACM.

Teodorovic, D., Lucic, P., Markovic, G., \& Orco, M. D. (2006). Bee colony optimization: Principles and applications. In Neural Network Applications in Electrical Engineering, 2006. NEUREL 2006. 8th Seminar on (pp. 151-156).

Tewari, A., Porter, C., Peabody, J., Crawford, E. D., Demers, R., Johnson, C. C., Wei, J. T., Divine, G. W., O'Donnell, C., Gamito, E. J., \& Menon, M. (2001). Predictive modeling techniques in prostate cancer. Molecular Urology, $5,147-152$. 
Thangavel, K., \& Manavalan, R. (2014). Soft computing models based feature selection for TRUS prostate cancer image classification. Soft Computing, 18, $1165-1176$.

Thompson, I. M., Ankerst, D. P., Chi, C., Goodman, P. J., Tangen, C. M., Lucia, cancer risk: Results from the prostate cancer prevention trial. Journal of the National Cancer Institute, 98, 529-534.

Tong, W., Xie, Q., Hong, H., Fang, H., Shi, L., Perkins, R., \& Petricoin, E. F. (2004). Using decision forest to classify prostate cancer samples on the basis of seldi-tof ms data: Assessing chance correlation and prediction confidence. Environmental Health Perspectives, 112, 1622-1627.

Took, C. C., \& Mandic, D. P. (2009). The quaternion lms algorithm for adaptive filtering of hypercomplex processes. IEEE Transactions on Signal Processing, 57, 1316-1327.

${ }_{1720}$ Tsamardinos, I., Brown, L., \& Aliferis, C. (2006). The max-min hill-climbing Bayesian network structure learning algorithm. Machine Learning, 65, 31-78.

Underwood, D. J., Zhang, J., Denton, B. T., Shah, N. D., \& Inman, B. A. (2012). Simulation optimization of psa-threshold based prostate cancer screening policies. Health Care Management Science, 15, 293-309.

1725 Vijaya, K., Khanna Nehemiah, H., Kannan, A., \& Bhuvaneswari, N. (2010). Fuzzy neuro genetic approach for predicting the risk of cardiovascular diseases. International Journal of Data Mining, Modelling and Management, 2, 388402.

Vos, P., Barentsz, J., Karssemeijer, N., \& Huisman, H. (2012). Automatic ${ }_{1730}$ computer-aided detection of prostate cancer based on multiparametric magnetic resonance image analysis. Physics in Medicine and Biology, 57, 1527.

Waljee, A., Higgins, P., \& Singal, A. (2014). A primer on predictive models. Clinical and Translational Gastroenterology, 5. 
Wang, Z., Gerstein, M., \& Snyder, M. (2009). RNA-Seq: a revolutionary tool for transcriptomics. Nature Reviews Genetics, 10, 57-63.

Xue, B., Zhang, M., Browne, W. N., \& Yao, X. (2016). A survey on evolutionary computation approaches to feature selection. IEEE Transactions on Evolutionary Computation, 20, 606-626.

Xutao, D., Huimin, G., \& Hesham, H. A. (2007). Cross-platform analysis of cancer biomarkers: A Bayesian Network approach to incorporating mass spectrometry and microarray data. Cancer Informatics, 3.

Yang, Z., Wu, A., \& Min, H. (2014). A multi-objective discrete pso algorithm based on enhanced search. In Intelligent Human-Machine Systems and Cybernetics (IHMSC), 2014 Sixth International Conference on (pp. 198-201). volume 2 .

Yu, E., Monaco, J. P., Tomaszewski, J., Shih, N., Feldman, M., \& Madabhushi, A. (2011). Detection of prostate cancer on histopathology using color fractals and probabilistic pairwise Markov models. In Engineering in Medicine and Biology Society, EMBC, 2011 Annual International Conference of the IEEE (pp. 3427-3430). IEEE.

Yu, Y., Zhang, J., Cheng, G., Schell, M., \& Okunieff, P. (2000). Multi-objective optimization in radiotherapy: applications to stereotactic radiosurgery and prostate brachytherapy. Artificial Intelligence in Medicine, 19, 39 - 51.

Zadeh, L. (1965). Fuzzy sets. Information and Control, 8, $338-353$. 